ACCepted to Ap.J.

Preprint typeset using $\mathrm{AT}_{\mathrm{E} X} \mathrm{X}$ style emulateapj v. 08/22/09

\title{
A SYSTEMATIC STUDY OF DEPARTURES FROM CHEMICAL EQUILIBRIUM IN THE ATMOSPHERES OF SUBSTELLAR MASS OBJECTS
}

\author{
Ivan HubenY $^{1}$ \& Adam Burrows ${ }^{1}$ \\ Accepted to Ap.J.
}

\begin{abstract}
We present a systematic study of the spectral consequences of departures from chemical equilibrium in the atmospheres of $\mathrm{L}$ and $\mathrm{T}$ dwarfs, and for even cooler dwarfs. The temperature/pressure profiles of the non-equilibrium models are fully consistent with the non-equilibrium chemistry. Our grid of non-equilibrium models includes spectra for effective temperatures from $200 \mathrm{~K}$ to $1800 \mathrm{~K}$, three surface gravities, four possible values of the coefficient of eddy diffusion in the radiative zone, and three different $\mathrm{CO} / \mathrm{CH}_{4}$ chemical reaction prescriptions. We also provide clear and cloudy model variants. We find, in keeping with previous studies, that there are essentially only two spectral regions where the effects of departures from chemical equilibrium can influence the predicted spectrum. These are in the $\mathrm{M}(\sim 4-5 \mu \mathrm{m})$ and $\mathrm{N}(8-14 \mu \mathrm{m})$ bands due to $\mathrm{CO}$ and $\mathrm{NH}_{3}$, respectively. The overabundance of CO translates into flux suppressions of at most $\sim 40 \%$ between effective temperatures of 600 and 1800 $\mathrm{K}$. The effect is largest around $T_{\text {eff }} \approx 1100 \mathrm{~K}$. The underabundance of ammonia translates into flux enhancements of no more than $\sim 20 \%$ for the $T_{\text {eff }}$ range from 300 to $1800 \mathrm{~K}$, with the largest effects at the lowest values of $T_{\text {eff }}$. The magnitude of the departure from chemical equilibrium increases with decreasing gravity, with increasing eddy diffusion coefficient, and with decreasing speed of the $\mathrm{CO} / \mathrm{CH}_{4}$ reaction. Though these effects are modest, they lead to better fits with the measured $\mathrm{T}$ dwarf spectra. Furthermore, the suppression in the $\mathrm{M}$ band due to non-equilibrium enhancements in the $\mathrm{CO}$ abundance disappears below $\sim 500 \mathrm{~K}$, and is only partial above $\sim 500 \mathrm{~K}$, preserving the $\mathrm{M}$ band flux as a useful diagnostic of cool atmospheres and maintaining its importance for searches for the cooler brown dwarfs beyond the Ts.
\end{abstract}

Subject headings: stars: abundances - stars: atmospheres - stars: individual (Gliese 570D) —stars: low-mass, brown dwrafs

\section{INTRODUCTION}

It has been long realized that departures from the local chemical equilibrium (LCE) may play a significant role in the atmospheres of the substellar-mass objects (SMO), that is of giant planets and brown dwarfs. Essentially, some chemical reactions occurring in these atmospheres may be very slow, so that vertical transport via convective motions or eddy diffusion can lead to departures from LCE. The mechanism was first suggested to operate in the Jovian planets of the solar system (Prinn \& Barshay 1977, Barshay \& Lewis 1978, Fegley \& Prinn 1985, Yung et al. 1988, and Fegley \& Lodders 1994).

Fegley \& Lodders (1996) suggested that the same mechanism can also operate in the atmospheres of brown dwarfs. Noll, Geballe, \& Marley (1997) studied in more detail the non-equilibrium enhancement of $\mathrm{CO}$ in the atmosphere of Gl 229B. Subsequently, Griffith \& Yelle (1999) and Saumon et al. (2000) provided a more detailed analysis. The latter paper also suggested that a non-equilibrium depletion of $\mathrm{NH}_{3}$ may be important. Saumon et al. (2003) demonstrated the effects of nonequilibrium chemistry for a range of effective temperatures ( $T_{\text {eff }}$ between 800 and $\left.1600 \mathrm{~K}\right)$. Recently, Saumon et al. $(2006,2007)$ showed that the non-equilibrium effects in the carbon and nitrogen chemistry have significant effects on the predicted spectrum of brown dwarfs, and in turn fit the observed spectrum of the T7.5 dwarf Gliese

\footnotetext{
${ }^{1}$ Department of Astronomy and Steward Observatory, The University of Arizona, Tucson, AZ 85721
}

570D (Saumon et al. 2006) and 2MASS 0415 (Saumon et al. 2007) much better than models based on local chemical equilibrium. Finally, Leggett et al. (2007) extended the Saumon et al. (2006) models to a larger range of parameters, and presented a comparison of model predictions with observed infrared colors.

In this paper, we extend and generalize the Saumon et al. and Leggett et al. treatments by computing a large grid of solar-metallicity models for effective temperatures $T_{\text {eff }}$ covering the whole domain of $\mathrm{T}$ and $\mathrm{L}$ dwarfs $(700$ - $1800 \mathrm{~K})$, as well as for cooler dwarfs $(200-700 \mathrm{~K})$, for three values of surface gravity $(\log g=4.5,5.0,5.5$ $\mathrm{cm} \mathrm{s}^{-2}$, for several values of the coefficient of eddy diffusion (see \$3.1), and for three different reaction rate timescales for the carbon reactions (see §3.1). We compute self-consistent models in the sense that the atmospheric structure ( $T / P$ profile) is computed taking into account departures from chemical equilibrium. We have computed two grids of models, one cloudless, and one including clouds composed of silicate condensates.

In $\oint 2$, we outline our procedures for computing model atmospheres, while allowing for departures from chemical equilibrium. In $₫ 3$, we present and analyze the predicted spectra computed with and without chemical equilibrium for $\mathrm{L}$ and $\mathrm{T}$ dwarfs. We explore the dependence on $T_{\mathrm{eff}}$, gravity, and eddy mixing coefficient in the radiative zone and compare clear and representative cloudy models. We also discuss the effect of non-equilibrium chemistry for objects cooler than the coolest $\mathrm{T}$ dwarfs down to effective temperatures of $200 \mathrm{~K}$. Then, in 4 , we briefly present a 
comparison between theory and observation in the midIR for Gliese 570D. Finally, in 55, we summarize our conclusions.

\section{MODELING PROCEDURES}

\subsection{Non-equilibrium Carbon and Nitrogen Chemistry}

The chemistry of carbon and nitrogen in the atmospheres of SMOs is essentially described by the net reactions

$$
\mathrm{CO}+3 \mathrm{H}_{2} \longleftrightarrow \mathrm{CH}_{4}+\mathrm{H}_{2} \mathrm{O}
$$

for carbon, and

$$
\mathrm{N}_{2}+3 \mathrm{H}_{2} \longleftrightarrow 2 \mathrm{NH}_{3}
$$

for nitrogen. Because of the strong $\mathrm{C}=\mathrm{O}$ and $\mathrm{N} \equiv \mathrm{N}$ bonds, the reactions (11) and (2) proceed much faster from right to left than from left to right. For instance, for carbon the reaction in which $\mathrm{CO}$ is converted to $\mathrm{CH}_{4}$ is very slow, and, therefore, $\mathrm{CO}$ can be vertically transported in the atmosphere to the upper and cooler regions by convective motions or eddy diffusion. The net result is an overabundance of $\mathrm{CO}$ and $\mathrm{N}_{2}$ and an underabundance of $\mathrm{NH}_{3}$ and $\mathrm{CH}_{4}$ in the upper atmosphere. Since more oxygen atoms are now tied in $\mathrm{CO}$, the mixing ratio of water is also reduced because of conservation of the total number of oxygen atoms.

The mixing (vertical transport) timescale, $t_{\mathrm{mix}}$, was suggested by Griffith \& Yelle (1999) to be parameterized by

$$
t_{\text {mix }}=H^{2} / K_{z z},
$$

where $H$ is the pressure scale height, and $K_{z z}$ is the coefficient of eddy diffusion. Equation (3) applies in the radiative (convectively stable) zone. In the convection zone, the mixing time is given by (Saumon et al. 2006):

$$
t_{\text {mix }}=3 H_{c} / v_{c},
$$

where $H_{c}$ is the convective mixing length (adopted here to be equal to $H$ ), and $v_{c}$ is the convective velocity. We stress that while the mixing time is well defined in the convection zone, it can only be roughly parameterized in the radiative zone. As discussed e.g. by Saumon et al. $(2006,2007)$, the reasonable values of $K_{z z}$ are in the range $10^{2}-10^{6} \mathrm{~cm}^{2} \mathrm{~s}^{-1}$.

The chemical reaction timescale, $t_{\text {chem }}$, generally decreases rapidly with increasing pressure and temperature. In the atmospheric regions where $t_{\text {mix }} \geq t_{\text {chem }}$, the species acquire their equilibrium mixing ratios. This occurs at temperatures and pressures larger than that at the depth where $t_{\text {mix }}=t_{\text {chem }}$. At smaller pressures, the species acquire mixing ratios equal to those at the $t_{\text {mix }}=t_{\text {chem }}$ layer, as first suggested for the case of the $\mathrm{CO} / \mathrm{CH}_{4}$ reaction by Prinn \& Barshay (1977).

The non-equilibrium mixing ratios thus depend sensitively on the chemical reaction timescales, which unfortunately are poorly known. For the $\mathrm{N}_{2} \rightarrow \mathrm{NH}_{3}$ conversion, we adopt the timescale given by Lodders \& Fegley (2002):

$$
\begin{array}{r}
t_{\text {chem }} \equiv t_{\mathrm{N}_{2}}=\frac{1}{\kappa_{\mathrm{N}_{2}} N\left(\mathrm{H}_{2}\right)} \\
\kappa_{\mathrm{N}_{2}}=8.54 \times 10^{-8} \exp \left(-\frac{81515}{T}\right),
\end{array}
$$

where $\kappa_{\mathrm{N}_{2}}$ is the rate constant for the $\mathrm{N}_{2} \rightarrow \mathrm{NH}_{3}$ reaction, and $N\left(\mathrm{H}_{2}\right)$ is the number density of $\mathrm{H}_{2}$.

The reaction timescales for the $\mathrm{CO} \rightarrow \mathrm{CH}_{4}$ conversion are uncertain because the correct chemical pathway is still unknown. We will, therefore, use three different timescales, suggested earlier in the literature:

i) the "slow" chemical timescale after Prinn \& Barshay (1977):

$$
\begin{gathered}
t_{\text {chem }} \equiv t_{\mathrm{CO}}=\frac{N(\mathrm{CO})}{\kappa_{\mathrm{CO}} N\left(\mathrm{H}_{2}\right) N\left(\mathrm{H}_{2} \mathrm{CO}\right)} \\
\kappa_{\mathrm{CO}}=2.3 \times 10^{-10} \exp \left(-\frac{36200}{T}\right),
\end{gathered}
$$

where $N(\mathrm{M})$ is the number density of species $\mathrm{M}$. ii) and iii) the "fast" chemical timescale after Yung et al. (1988) or Dunning et al. (1984). In both cases the timescale is given by

$$
t_{\mathrm{CO}}=\frac{N(\mathrm{CO})}{\kappa_{7} N(\mathrm{H}) N\left(\mathrm{H}_{2} \mathrm{CO}\right)},
$$

where the rate coefficient differs in each reference. We use tabulated values presented in Yung et al. (1988 - their Table III), and interpolate to current temperatures. In this paper, we call the Dunning et al. timescale "fast1," and the Yung et al. timescale "fast2." As we shall show in $\S 3$, these two fast timescales produce very similar results.

\subsection{Atmosphere and Spectrum Modeling}

We use the updated code CoolTLUSTY, described in Sudarsky, Burrows, \& Hubeny (2003), Hubeny, Burrows, \& Sudarsky (2003), and Burrows, Sudarsky, \& Hubeny (2006 - BSH), which is a variant of the universal atmospheric code TLUSTY (Hubeny 1988; Hubeny \& Lanz 1995). CoOLTLUSTY solves self-consistently a set of radiative transfer equations for selected frequency points (typically 5000 points logarithmically spaced between $7 \times 10^{14} \mathrm{~Hz}$ and $10^{12} \mathrm{~Hz}$ ), and the equation of radiative + convective equilibrium. The atmosphere is assumed to be in hydrostatic equilibrium. The opacities are not computed on the fly; instead, they are interpolated from pre-calculated opacity tables for the current values of temperature and density. Our opacity database is described in detail by Sharp \& Burrows (2007). The atmospheric structure is computed iteratively, applying the Newton-Raphson method (first applied in the case of stellar atmospheres by Auer \& Mihalas 1969 with the name "complete linearization"). The discretized and linearized equations are re-organized in the so-called Rybicki scheme (Mihalas 1978), which renders the computer time linearly proportional to the number of frequencies (and not proportional to the cube of the number of frequencies as in the case of the original complete linearization). This allows us to avoid using the accelerated lambda iteration (ALI) scheme to treat the radiative transfer equation, which leads to faster and more stable convergence, without compromising on computer time.

We have found that the most stable procedure to compute non-LCE models is to perform several iterations (typically 6) of the linearization method assuming chemical equilibrium (using the algorithm of Burrows \& Sharp 1999 to determine the equilibrium abundances of all 
species), and then to switch the non-LCE algorithm. In each subsequent iteration, one calculates the intersection point where the mixing time for the current $T / P$ profile equals the reaction time (computed again for the current $T / P$ profile); the number densities of $\mathrm{CO}, \mathrm{CH}_{4}, \mathrm{H}_{2} \mathrm{O}$, and $\mathrm{NH}_{3}$ are set to constant values equal to those found at the intersection points for pressures lower than the pressure at the intersection. The opacities in these regions are modified accordingly. The next iteration of the linearization scheme is then performed with the new opacities. The overall process usually converges in a few additional iterations.

When clouds are taken into account, we employ the scheme described in BSH. In the case of L and T dwarfs, we represent individual cloud decks of many condenstate species by a single extended cloud deck of one representative condenstate. As in BSH, we take forsterite as a representative condensate, and assume the cloud deck to extend between the intersection of the condensation curve of forsterite with the current $T / P$ profile, and the pressure where the local temperature equals $2300 \mathrm{~K}$ (which schematically represents the condensation curve of the most refractory condenstate), and with exponential decreases of particle density on both sides of the cloud. In the terminology of BSH, this is an E-type cloud. In all the present models, we assume the modal particle size $100 \mu \mathrm{m}$.

\section{RESULTS}

\subsection{L and T Dwarfs}

Our effort has generated an extensive grid of nonequilibrium model spectra for effective temperatures from $700 \mathrm{~K}$ to $1800 \mathrm{~K}$ (in steps of $100 \mathrm{~K}$ ), for three values of surface gravity, $g=10^{4.5}, 10^{5.0}$, and $10^{5.5} \mathrm{~cm} \mathrm{~s}^{-2}$, for four values of the coefficient of eddy diffusion in the radiative zone $K_{z z}=10^{2}, 10^{4}, 10^{6}$, and $10^{8} \mathrm{~cm}^{2} \mathrm{~s}^{-1}$, and for three different $\mathrm{CO} / \mathrm{CH}_{4}$ reaction time prescriptions ${ }^{2}$. We have also provided clear and cloudy model variants. The latter assumes an E-type forsterite cloud with a modal particle size of $100 \mu \mathrm{m}$. In the present paper, we consider only solar-composition models, where the solar elemental abundances are from Asplund, Grevesse, \& Sauval (2006).

We will first discuss composition profiles and their trends with effective temperature, surface gravity, diffusion coefficient, and the $\mathrm{CO} / \mathrm{CH}_{4}$ reaction speed. Figure 11displays the composition profiles of the non-equilibrium species, $\mathrm{CO}, \mathrm{CH}_{4}, \mathrm{NH}_{3}$, and $\mathrm{H}_{2} \mathrm{O}$, as a function of the local temperature in the atmosphere, for a representative model with $T_{\text {eff }}=900 \mathrm{~K}, g=10^{5.5} \mathrm{~cm} \mathrm{~s}^{-2}$, and $K_{z z}=10^{4} \mathrm{~cm}^{2} \mathrm{~s}^{-1}$. The full lines show the nonequilibrium mole fractions or, equivalently, the number fractions (expressed as a fraction of the total number of particles), while the dashed lines show the equilibrium number fractions. For this model, the non-equilibrium effects in water and methane are negligible. The nonequilibrium number fraction of ammonia is smaller than the equilibrium one for $T<1200 \mathrm{~K}$, as first shown by Saumon et al. (2006). The non-equilibrium mixing ratio of $\mathrm{CO}$ is significantly larger than the equilibrium one, which in turn leads to a significant strengthening

2 Electronic versions of these spectral models can be found online at http://zenith.as.arizona.edu// burrows. of the $4.7-\mu \mathrm{m}$ feature (and, to a lesser extent, to other $\mathrm{CO}$ features at 3.4 and $2.8 \mu \mathrm{m}$ ) in non-equilibrium models. Moreover, the non-equilibrium mixing ratio of $\mathrm{CO}$ is larger for the slower $\mathrm{CO} / \mathrm{CH}_{4}$ reaction, because the intersection of the $t_{\text {mix }}(T)$ curve with the $t_{\mathrm{CO}}(T)$ curve occurs deeper in the atmosphere for higher temperature, at which the equilibrium $\mathrm{CO}$ mixing ratio is higher (see also Fig. (5). Figure 1 is analogous to Fig. 3 of Saumon et al. (2006).

To depict the trend of composition profiles with effective temperature, we display in Fig. 2 composition profiles as in Fig. 1, but for $T_{\text {eff }} / 100 \mathrm{~K}=8,10,12,14,16$, and 18. To avoid cluttering the figure, the models are not labeled by the values of $T_{\text {eff }}$. Instead, the models are distinguished by the fact that the higher the effective temperature is, the longer the curves continue toward high temperatures (i.e., to the right side of the plot). First, we stress that the equilibrium abundance patterns follow from the basic chemistry (Burrows \& Sharp 1999; Lodders \& Fegley 2002), and are well understood. Briefly, the same local temperature is reached at lower pressure for models with higher effective temperatures (see Fig. 7 for an explicit demonstration). Due to Le Chatelier's principle, at a given local temperature the abundance of $\mathrm{CH}_{4}$ decreases and the abundance of $\mathrm{CO}$ increases with increasing $T_{\text {eff }}$. Analogously, the abundance of $\mathrm{NH}_{3}$ decreases, and the abundance of $\mathrm{N}_{2}$ (not shown here) increases with increasing $T_{\text {eff }}$. These effects are clearly shown in Fig. 2,

For all models displayed here, the non-equilibrium effects in water and methane are negligible. The nonequilibrium abundance of $\mathrm{CO}$ is always larger than the equilibrium abundance for $T \stackrel{\sim}{<} 1000 \mathrm{~K}$. For very low $T_{\text {eff }}$, although the non-equilibrium abundance of $\mathrm{CO}$ is significantly larger than the equilibrium abundance, it is still small in absolute value, and, therefore, does not have much of an effect on the model structure and predicted spectra. For the highest $T_{\text {eff }}$, the equilibrium abundance profile of $\mathrm{CO}$ becomes flatter, and, consequently, the differences between the non-equilibrium and equilibrium abundance profiles of $\mathrm{CO}$ decrease, and so do the differences in the predicted spectra. This is demonstrated in Fig. 8. For ammonia, departures from equilibrium become smaller for higher effective temperatures, because the equilibrium ammonia composition profile becomes flat. Therefore, fixing the ammonia number fraction at the intersection of the $t_{\text {mix }}$ and the $t_{\mathrm{NH}_{3}}$ curves does not lead to a significant difference in the composition. Therefore, the non-equilibrium effects in ammonia, and, consequently, in the predicted flux in the $8-14 \mu \mathrm{m}$ region, decrease with increasing $T_{\text {eff }}$. This also is demonstrated in Fig. 8. Moreover, because of its diminished number fraction, ammonia becomes a less important source of opacity as the temperature increases.

In Fig. 3. we show the trend of the composition profiles with the surface gravity, for $T_{\text {eff }}=900 \mathrm{~K}, K_{z z}=10^{4} \mathrm{~cm}^{2}$ $\mathrm{s}^{-1}$, and the fast $\mathrm{CO} / \mathrm{CH}_{4}$ reaction. The intersection of the $t_{\mathrm{mix}}$ and the $t_{\mathrm{CO}}$ curves occurs at essentially the same temperature for all three surface gravities (see Fig. (4). However, at a given temperature, the model with the lowest gravity exhibits the lowest pressure, and, therefore, the $\mathrm{CO}$ mixing ratio at the intersection is higher for the lower-gravity models. Consequently, the effects 
of departures from chemical equilibrium are greater for the low-gravity models.

Figure 4 displays the mixing time and the reaction times for the models displayed in Fig. 3. Notice the abrupt change of the mixing time at $T \approx 1800 \mathrm{~K}$; this corresponds to the onset of convection, where the mixing times are about five orders of magnitude faster. To explain the behavior of models with different coefficients of eddy diffusion, and with different reaction timescales for the $\mathrm{CO} / \mathrm{CH}_{4}$ reaction, we display in Fig. [5] the mixing times for four different diffusion coefficients, $K_{z z}=$ $10^{2}, 10^{4}, 10^{6}$, and $10^{8} \mathrm{~cm}^{2} \mathrm{~s}^{-1}$. By increasing the coefficient of eddy diffusion, $K_{z z}$, the mixing time decreases and, consequently, the intersection of the chemical reaction timescale curves and the mixing time curve occurs at higher temperatures. Therefore, the non-equilibrium mixing ratio of $\mathrm{CO}$ is higher. For the $\mathrm{N}_{2} / \mathrm{NH}_{3}$ reaction, and for $K_{z z}>10^{4} \mathrm{~cm}^{2} \mathrm{~s}^{-1}$, the chemical time curve intersects the mixing time curve in the convection zone at very high temperature $(T \approx 2100 \mathrm{~K})$.

In Fig. 6, we display trends of the chemical reaction time with the effective temperature. The $\mathrm{N}_{2} / \mathrm{NH}_{3}$ reaction timescale is relatively insensitive to the model effective temperature, while for the $\mathrm{CO} / \mathrm{CH}_{4}$ reaction, the intersection of the curves moves to higher temperatures and pressures for higher effective temperatures. It would seem at first sight that the departures from chemical equilibrium would increase with increasing $T_{\text {eff }}$. However, as we have shown in Fig. 2, the abundance profile of $\mathrm{CO}$ becomes flatter and, thus, the departures from chemical equilibrium decrease instead.

In Fig. 7 we display the temperature/pressure profiles for selected models of our grid. The differences in the local temperature between the equilibrium and nonequilibrium models are not large, but nevertheless appreciable.

We now turn to emergent spectra. In Fig. 8, we display the emergent spectra for all effective temperatures, and for one representative value of $g=10^{5.5} \mathrm{~cm} \mathrm{~s}^{-2}$, for $K_{z z}=10^{4} \mathrm{~cm}^{2} \mathrm{~s}^{-1}$, and for the "slow" reaction rate of the $\mathrm{CO} / \mathrm{CH}_{4}$ reaction of Prinn \& Barshay (1977). There are essentially only two wavelength regions where the departures from chemical equilibrium influence the predicted spectrum, the region around 4.7 microns (due to non-equilibrium abundances of $\mathrm{CO}$ ), and a wide region between 8 and 14 microns, due to non-equilibrium abundances of $\mathrm{NH}_{3}$. The $\mathrm{CO}$ feature has its peak for effective temperatures between $800 \mathrm{~K}$ and $1200 \mathrm{~K}$; the effect generally decreases for hotter models and essentially disappears at $1800 \mathrm{~K}$. The non-equilibrium effect of ammonia already disappears for $T_{\text {eff }} \gtrsim 1200 \mathrm{~K}$. The behavior of the associated spectra is readily understood upon examination of the composition profiles displayed in Figs. 13 (see also the related discussion). Figure 8 is a synoptic display of the overall model trends, but is not meant to communicate details of the predicted spectra. Therefore, we later present in Figs. 11, 12, and 13 enlarged plots of the two non-equilibrium regions, the $\mathrm{CO}$ region between 3 and $5 \mu \mathrm{m}$, and the ammonia region between 8 and 14 $\mu \mathrm{m}$. In these and subsequent figures that display the predicted spectra, we smooth the the computed spectrum by a 10-point boxcar average, which corresponds to a resolution of about $1 / 100$. The corresponding models with clouds are displayed in Fig. 9 and behave analogously, thus demonstrating that the presence of clouds does not lead to any qualitative differences in the magnitude of departures from chemical equilibrium.

Figure 10 shows the sensitivity of the predicted spectrum to the surface gravity. The non-equilibrium effects generally increase with decreasing gravity, as was explained in the discussion of Fig. 3. Figure 11 presents a more detailed view of the $\mathrm{CO}$ non-equilibrium region between 3 and $5 \mu \mathrm{m}$, for one particular model with $T_{\text {eff }}=900 \mathrm{~K}$ and $g=10^{5.5} \mathrm{~g} \mathrm{~cm}^{-2}$. We display both the LCE model and four non-equilibrium models with $K_{z z}=10^{2}, 10^{4}, 10^{6}$, and $10^{8} \mathrm{~cm}^{2} \mathrm{~s}^{-1}$. All models are for the "slow" reaction rate of Prinn \& Barshay (1977) for the $\mathrm{CO} / \mathrm{CH}_{4}$ reaction. An analogous plot for the models with the "fast2" reaction of Yung et al. (1988) is displayed in Fig. 12. The magnitude of departures from LCE increases significantly with increasing $K_{z z}$. This is because the mixing time decreases with increasing $K_{z z}$ and, thus, the intersection of the $t_{\text {mix }}$ and the $t_{\mathrm{CO}}$ curves occurs deeper in the atmosphere, where the mixing ratio of CO is larger. This was already demonstrated in Fig. 5 , As Fig. 12] shows, the departures from LCE are smaller for the faster $\mathrm{CO} / \mathrm{CH}_{4}$ reaction rate, as demonstrated previously in Fig. 1

In Fig. 13. we display a more detailed view of the ammonia region between 8 and $14 \mu \mathrm{m}$, for the same models as displayed in Fig. 12. The departures from chemical equilibrium are essentially the same as predicted by Saumon et al. $(2006,2007)$. Unlike the case of CO, the abundance of ammonia and, therefore, the flux in the region between 8 and $14 \mu \mathrm{m}$, is relatively insensitive to the value of $K_{z z}$. This is because the abundance profile of ammonia is rather flat (see Figs. 11- 3), and, therefore, the depth of intersection of the $t_{\mathrm{N}_{2}}$ and the $t_{\text {mix }}$ curves does not matter very much. The departures of ammonia from LCE are largely insensitive to the speed of the $\mathrm{CO} / \mathrm{CH}_{4}$ reaction, as expected. We do not show this in the figure because the corresponding plots are essentially identical. The predicted departures of $\mathrm{NH}_{3}$ from LCE seem to be quite robust.

To better display departures from chemical equilibrium, we plot in Fig. 14 the ratio of the integrated flux between $4.4 \mu \mathrm{m}$ and $5 \mu \mathrm{m}$ of the non-equilibrium and equilibrium models, as a function of effective temperature. The upper panel displays the dependence of the non-equilibrium ratio for $g=10^{5} \mathrm{~cm} \mathrm{~s}^{-2}$, for the "slow" $\mathrm{CO} / \mathrm{CH}_{4}$ reaction, and the "fast2" $\mathrm{CO} / \mathrm{CH}_{4}$ reaction. Each set of models is computed for three values of $K_{z z}$. The curves are labelled by the value of $\log _{10} K_{z z}$ in $\mathrm{cm}^{2}$ $\mathrm{s}^{-1}$. As discussed above, assuming the "slow" $\mathrm{CO} / \mathrm{CH}_{4}$ reaction increases the departures from LCE significantly, reaching about $30 \%$ at $T_{\text {eff }}=1100 \mathrm{~K}\left(\right.$ at $K_{z z}=10^{4}$ $\mathrm{cm}^{2} \mathrm{~s}^{-1}$ ), and decreasing toward higher temperatures. For the "slow2" reaction, the maximum effect is around $T_{\text {eff }}=1300 \mathrm{~K}$, where it reaches about $10 \%$ (again, at $K_{z z}=10^{4} \mathrm{~cm}^{2} \mathrm{~s}^{-1}$ ). The lower panel displays the dependence of the non-equilibrium ratio on surface gravity for two sets of models. In all models, $K_{z z}=10^{4} \mathrm{~cm}^{2} \mathrm{~s}^{-1}$. The upper set of models (diamonds connected by dashed lines) are for the "fast2" $\mathrm{CO} / \mathrm{CH}_{4}$ reaction, while the lower set of models (stars connected by dotted lines) are for the "slow" $\mathrm{CO} / \mathrm{CH}_{4}$ reaction. The curves are labelled 
by the value of $\log _{10} g$ in $\mathrm{cm} \mathrm{s}^{-2}$. As discussed above, the lower-gravity models exhibit larger departures from equilibrium for temperature below $1300 \mathrm{~K}$. However, the trend is reversed for $T_{\text {eff }}>1300 \mathrm{~K}$, where the lower gravity models are closer to equilibrium.

\subsection{Beyond the T Dwarfs}

In this section, we briefly explore the effects on spectra of departures from chemical equilibrium for brown dwarfs cooler than the coolest known T dwarfs (see also Burrows, Sudarsky, \& Lunine 2003). To this end, we extend the grid of models described in 3.1 and compute models for $T_{\text {eff }}=200,300,400,500$, and $600 \mathrm{~K}$. For the models below $500 \mathrm{~K}$, we include the effect of a water cloud with a modal particle size of $100 \mu \mathrm{m}$ and a super-saturation parameter of $1.0 \%$. The other basic parameters are the same as in 33.1. In Fig. 16, we display the mixing time and the reaction times for three models with $T_{\text {eff }}=200,400$, and $600 \mathrm{~K}$, for $g=10^{5} \mathrm{~cm} \mathrm{~s}^{-2}$, $K_{z z}=10^{4} \mathrm{~cm}^{2} \mathrm{~s}^{-1}$, and the "fast2" $\mathrm{CO} / \mathrm{CH}_{4}$ reaction. In all models, the intersection of the mixing time and the reaction times occurs in the convection zone. Therefore, the actual value of the diffusion coefficient $K_{z z}$, which influences the mixing time only at temperatures and pressures lower than those at the intersection, is inconsequential. (In other words, $K_{z z}$ influences the $T_{\operatorname{mix}}$ curve only on the left of the intersection point, which is already determined by the local value of the convective velocity.) Note that the intersections move to lower temperature with decreasing $T_{\text {eff }}$. The somewhat wiggly character of the $t_{\text {mix }}$ curve in convection zone follows form the fact the the convective velocity is given by (e.g., Mihalas 1978)

$$
v=(g Q H / 8)^{1 / 2}\left(\nabla-\nabla_{E}\right)(l / H)^{2},
$$

where $g$ is the surface gravity, $H$ the pressure scale height, $Q=(\partial \ln \rho / \partial \ln T)_{P}, l$ is the mixing length, and $\left(\nabla-\nabla_{E}\right)$ is a difference between the true temperature gradient, $\nabla=\partial \ln T / \partial \ln P$, and the gradient of the convective elements. This is computed by considering the efficiency of convective transport. In the standard mixinglength approach, the efficiencies are computed differently for Rosseland optical depth smaller and larger than unity. The wiggly behavior corresponds to a transition between Rosseland optical depth larger than and smaller than 1. In any case, the uncertainty in the exact value of the convective velocity does not influence the non-equilibrium abundances of $\mathrm{CO}, \mathrm{CH}_{4}$, and $\mathrm{NH}_{3}$ in a significant way.

Figure 17depicts the abundance profiles for the models displayed in Fig. 16. The non-equilibrium number fraction of CO sharply decreases mixing time with decreasing $T_{\text {eff }}$. This is a consequence of the fact that while the intersection points of the $t_{\text {mix }}$ and the $t_{\mathrm{CO}}$ curves occur at similar temperatures around $1100 \mathrm{~K}$, these intersection points are reached at much higher pressures for lower $T_{\text {eff }}$. Consequently, the equilibrium number fraction of $\mathrm{CO}$ at the intersection point is lower. For ammonia, the equilibrium abundance profiles become flatter for deceasing $T_{\text {eff }}$ and, therefore, the differences between the equilibrium and non-equilibrium number fractions are smaller.

In Fig. 18, we depict the predicted flux for the three models displayed in Figs. 16 and 17. The hottest model, at $T_{\text {eff }}=600 \mathrm{~K}$, exhibits the same features as late $\mathrm{T}$ dwarfs (see 3.1 ), namely the non-equilibrium flux is higher in the $8-14 \mu \mathrm{m}$ region (by about $15-20 \%$ ) and smaller in the the $4-5 \mu \mathrm{m}$ region (on average, by about $10 \%)$. In the cooler model at $T_{\text {eff }}=400 \mathrm{~K}$, the departures in the $8-14 \mu \mathrm{m}$ region due to non-equilibrium chemistry of ammonia are slightly smaller than in the $600-\mathrm{K}$ model. However, the differences in the $4-5 \mu \mathrm{m}$ region essentially disappear because the non-equilibrium mixing ratio of $\mathrm{CO}$, though much higher than the equilibrium one, is already quite small. Therefore, $\mathrm{CO}$ contributes but little to the opacity in this region. Finally, for the coolest model at $T_{\text {eff }}=200 \mathrm{~K}$, the non-equilibrium effects almost disappear in both the $4-5 \mu \mathrm{m}$ and $8-14 \mu \mathrm{m}$ regions.

To diagnose the trends described above, we display in Fig. 19 the temperature/pressure profiles for the same models (both non-equilibrium and equilibrium) depicted in Figs. 16 - 18. In Fig. 20, we display the "radiation formation temperature" as a function of wavelength for the two extreme models with $T_{\text {eff }}=200$ and $600 \mathrm{~K}$. The radiation formation temperature is defined as a local temperature in the atmosphere where the monochromatic optical depth is $2 / 3$. This quantity indicates the position/layer from which a photon of a given wavelength effectively decouples from the atmosphere.

\section{MODEL COMPARISON WITH GLIESE 570D OBSERVATIONS}

In Fig. 21, we display the observed spectrum of Gliese $570 \mathrm{D}$, kindly supplied to us in digital form by M. Cushing (Cushing et al. 2006). This is one of the latest known $\mathrm{T}$ dwarfs, and is the object studied by Saumon et al. (2006). We also display two theoretical models computed for $T_{\text {eff }}=800 \mathrm{~K}$ and $g=10^{5} \mathrm{~cm} \mathrm{~s}^{-2}$, namely an LCE and a non-LCE model. The non-equilibrium model is computed with $K_{z z}=10^{4} \mathrm{~cm}^{2} \mathrm{~s}^{-1}$, and for the "fast2" $\mathrm{CO} / \mathrm{CH}_{4}$ reaction rate, but, as demonstrated in Fig. 13, this predicted flux essentially coincides with the flux computed for all other values of $K_{z z}$ and $\mathrm{CO} / \mathrm{CH}_{4}$ reaction time prescriptions. The agreement between the observed and predicted spectrum for non-LCE model is excellent in the region between 8 and $14 \mu \mathrm{m}$, while the fit is much worse for the LCE model. We thus confirm the results of Saumon et al. ${ }^{3}$. In a future work, we will show comparisons between our non-equilibrium spectral models and observations in the near- and mid-IR of other late $\mathrm{T}$ dwarfs.

\section{DISCUSSION AND CONCLUSIONS}

In this paper, we have performed a systematic study of the spectral consequences of departures from chemical equilibrium in carbon and nitrogen chemistry in the atmospheres of $\mathrm{L}$ dwarfs, $\mathrm{T}$ dwarfs, and substellarmass objects down to $200 \mathrm{~K}$. Unlike previous investigations, the temperature/pressure profiles of the nonequilibrium models are fully consistent with the nonequilibrium chemistry. Such models reveal that failure to calculate the non-equilibrium atmospheric profiles in a self-consistent fashion can result in errors of as much as $\sim 50-100 \mathrm{~K}$ in the atmospheric temperatures at a given pressure. Our effort has generated an extensive grid of

3 Note that the predicted fluxes for both our models and those of Saumon et al. (2006) are too low between 7 and $8 \mu \mathrm{m}$, for both LCE and non-LCE models. The reason for this is unclear, but may be related to deficiencies in the extant water opacity databases. 
non-equilibrium model spectra for effective temperatures from $700 \mathrm{~K}$ to $1800 \mathrm{~K}$ (in steps of $100 \mathrm{~K}$ ), for three values of surface gravity, $g=10^{4.5}, 10^{5.0}$, and $10^{5.5} \mathrm{~cm} \mathrm{~s}^{-2}$, for four values of the coefficient of eddy diffusion in the radiative zone $K_{z z}=10^{2}, 10^{4}, 10^{6}$, and $10^{8} \mathrm{~cm}^{2} \mathrm{~s}^{-1}$, and for three different $\mathrm{CO} / \mathrm{CH}_{4}$ reaction time prescriptions. We have also provided clear and cloudy model variants. We find, in keeping with previous studies, that there are essentially only two regions where the effects of departures from chemical equilibrium significantly influence the predicted spectrum. These are in the $\mathrm{M}(\sim 4-5 \mu \mathrm{m})$ and $\mathrm{N}$ $(8-14 \mu \mathrm{m})$ bands due to $\mathrm{CO}$ and $\mathrm{NH}_{3}$, respectively. The overabundance of $\mathrm{CO}$ can be very large, but translates into flux suppressions in the $\mathrm{M}$ band of at most $\sim 40 \%$ between effective temperatures of 800 and $1800 \mathrm{~K}$. The effect is largest around $T_{\text {eff }} \approx 1100 \mathrm{~K}$. The underabundance of ammonia can be significant, but translates into flux enhancements in the $\mathrm{N}$ band of no more than $\sim 20 \%$ for the $T_{\text {eff }}$ range from 700 to $1800 \mathrm{~K}$, with the largest effects at the lowest values of $T_{\text {eff }}$. The magnitude of the departure from chemical equilibrium increases with decreasing gravity, with increasing eddy diffusion coefficient, and with decreasing speed of the $\mathrm{CO} / \mathrm{CH}_{4}$ reaction. Though these effects are modest, they lead to better fits with the measured $\mathrm{T}$ dwarf spectra (see also Saumon et al. 2006,2007).

In addition, we have calculated an exploratory sequence of non-equilibrium models down to $200 \mathrm{~K}$, including the possible effects of water cloud formation. These models show that in the region "beyond the $\mathrm{T}$ dwarfs"
(Burrows, Sudarsky, \& Lunine 2003) the suppression effect of non-equilibrium $\mathrm{CO}$ in the $\mathrm{M}$ band diminishes, then disappears, with decreasing $T_{\text {eff }}$.

Hence, despite the modest suppression in the $\mathrm{M}$ band due to non-equilibrium $\mathrm{CO}$ chemistry for $T_{\text {eff }} \mathrm{s}$ above $\sim 500 \mathrm{~K}$, non-equilibrium effects do not compromise the use of the $\mathrm{M}$ band flux as a useful diagnostic of cool atmospheres and as one of their most prominent features. At the higher $T_{\text {eff }}$ s above $\sim 500 \mathrm{~K}$, this is due in part to the saturation of the augmented $\mathrm{CO}$ absorption, but importantly it is due to the fact that only about half of the $\mathrm{M}$ band overlaps with the $4.7-\mu \mathrm{m} \mathrm{CO}$ absorption feature. At lower $T_{\text {eff }}$ b below $\sim 400 \mathrm{~K}$, non-equilibrium effects in the $\mathrm{M}$ and $\mathrm{N}$ bands all but disappear. Overall, the prominence and importance of the $\mathrm{M}$ band flux for searches for the coolest dwarfs and for brown dwarfs ("Y" dwarfs ?) beyond the $\mathrm{T}$ dwarf range is not substantially affected by non-equilibrium chemistry.

We thank M. Cushing, J. Budaj, and W. Hubbard for fruitful discussions and help during the course of this work. We also thank the referee, K. Lodders, for her detailed comments. This study was supported in part by NASA grants NAG5-10760, NNG05GG05G, NNG04GL22G and NNX07AG80G, and through the NASA Astrobiology Institute under Cooperative Agreement No. CAN-02-OSS-02 issued through the Office of Space Science.

\section{REFERENCES}

Asplund, M., Grevesse, N., \& Sauval, A. J. 2006, Commun. in Asteroseismology, 147, 76

Auer, L. H., \& Mihalas, D. 1969, ApJ, 158, 641

Barshay, S. S., \& Lewis, J. S. 1978, Icarus, 33, 593

Burrows, A., \& Sharp, C. M. 1999, ApJ, 512, 843

Burrows, A., Sudarsky, D., \& Hubeny, I. 2006, ApJ, 640, 1063

Burrows, A., Sudarsky, D., \& Lunine, J.I. 2003, ApJ, 596, 587

Cushing, M. C. et al. 2006, ApJ, 648, 614

Dunning, T. H., Harding, L. B., \& Wagner, A. F. 1984, in Theory and Modeling for Material Designs, Texas A \& M University

Fegley, B., Jr., \& Prinn, R. G. 1985, ApJ, 299, 1067

Fegley, B., Jr., \& Lodders, K. 1996, ApJ, 472, L37

Griffith, C. A., \& Yelle, R. V. 1999, ApJ, 519, L85

Hubeny, I. 1988, Comput. Phys. Commun., 52, 103

Hubeny, I., Burrows, A., \& Sudarsky, D. 2003, ApJ, 594, 1011

Hubeny, I., \& Lanz, T. 1995, ApJ, 439, 875

Leggett, S. K., Saumon, D., Marley, M. S., Geballe, T. R., Golimowski, D. A., Stephens, D., \& Fan, X. 2007, ApJ, 655, 1079

Lodders, K., \& Fegley, B., Jr. 2002, Icarus, 155, 393

Mihalas, D. 1978, Stellar Atmospheres, 2nd ed., Freeman, San Francisco
Noll, K. S., Geballe, T. R., \& Marley, M.S. 1997, ApJ, 489, L87

Prinn, G. G., \& Barshay, S. S. 1977, Science, 198, 1031

Saumon, D., Geballe, T. R., Leggett, S. K., Marley, M. S., Freedman, R. S., Lodders, K., Fegley, B. Jr., \& Sengupta, S. K. 2000, ApJ, 541, 374

Saumon, D., Marley, M. S., Lodders, K., \& Freedman, R. S. 2003, in "Brown Dwarfs," IAU Symposium 211, 345

Saumon, D., Marley, M. S., Cushing, M. C., Leggett, S. K.,

Roellig, T. L., Lodders, K., \& Freedman, R. S. 2006, ApJ, 647, 552

Saumon, D., Marley, M. S., Leggett, S.K., Geballe, T.R., Stephens, D., Golimowski, D.A., Cushing, M. C., Fan, X., Rayner, J.T., Lodders, K., \& Freedman, R.S. 2007, ApJ, 656, 1136

Sharp, C. M., \& Burrows, A. 2007, ApJS, 168, 140

Sudarsky, D., Burrows, A., \& Hubeny, I. 2003, ApJ, 588, 1121

Yung, Y. L., Drew, W. A., Pinto, J. P., \& Friedl, R. R. 1988,

Icarus, 73,516 


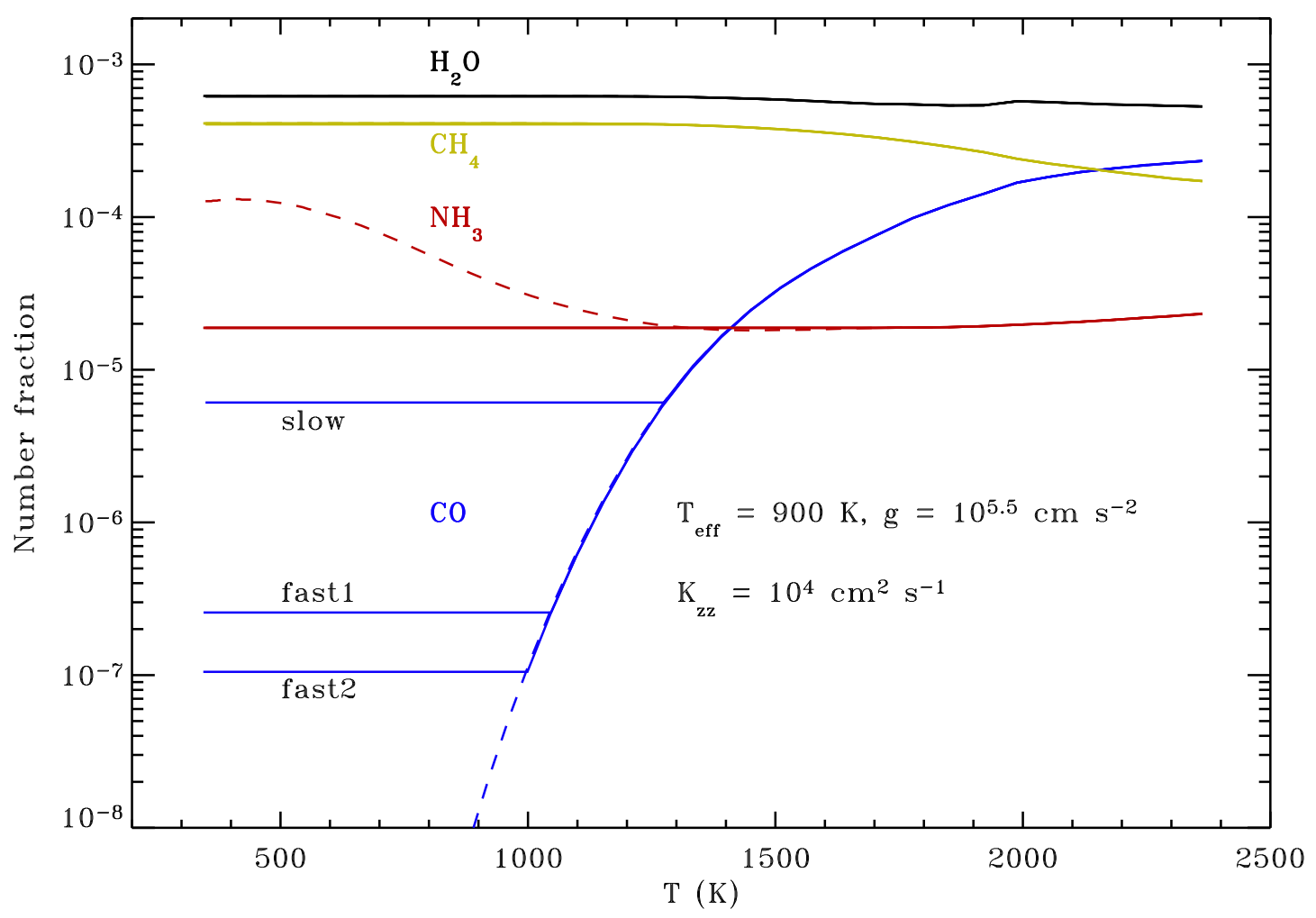

Fig. 1. - A comparison of the equilibrium (dashed lines) and non-equilibrium (solid lines) abundances of water (black), methane (yellow), ammonia (red), and CO (blue), for a representative model with $T_{\text {eff }}=900 \mathrm{~K}, g=10^{5.5} \mathrm{~cm} \mathrm{~s}^{-2}$, and $K_{z z}=10^{4} \mathrm{~cm}^{2} \mathrm{~s}^{-1}$. In this case, water and methane remain essentially at their equilibrium abundances, while ammonia becomes underabundant at the upper layers (leading to a lower opacity and, thus, higher predicted flux in the 8-14 $\mu \mathrm{m}$ region for non-equilibrium models). The plots also show that CO exhibits larger departures from equilibrium with the slow reaction. See Fig. [5] and the text for a discussion. 


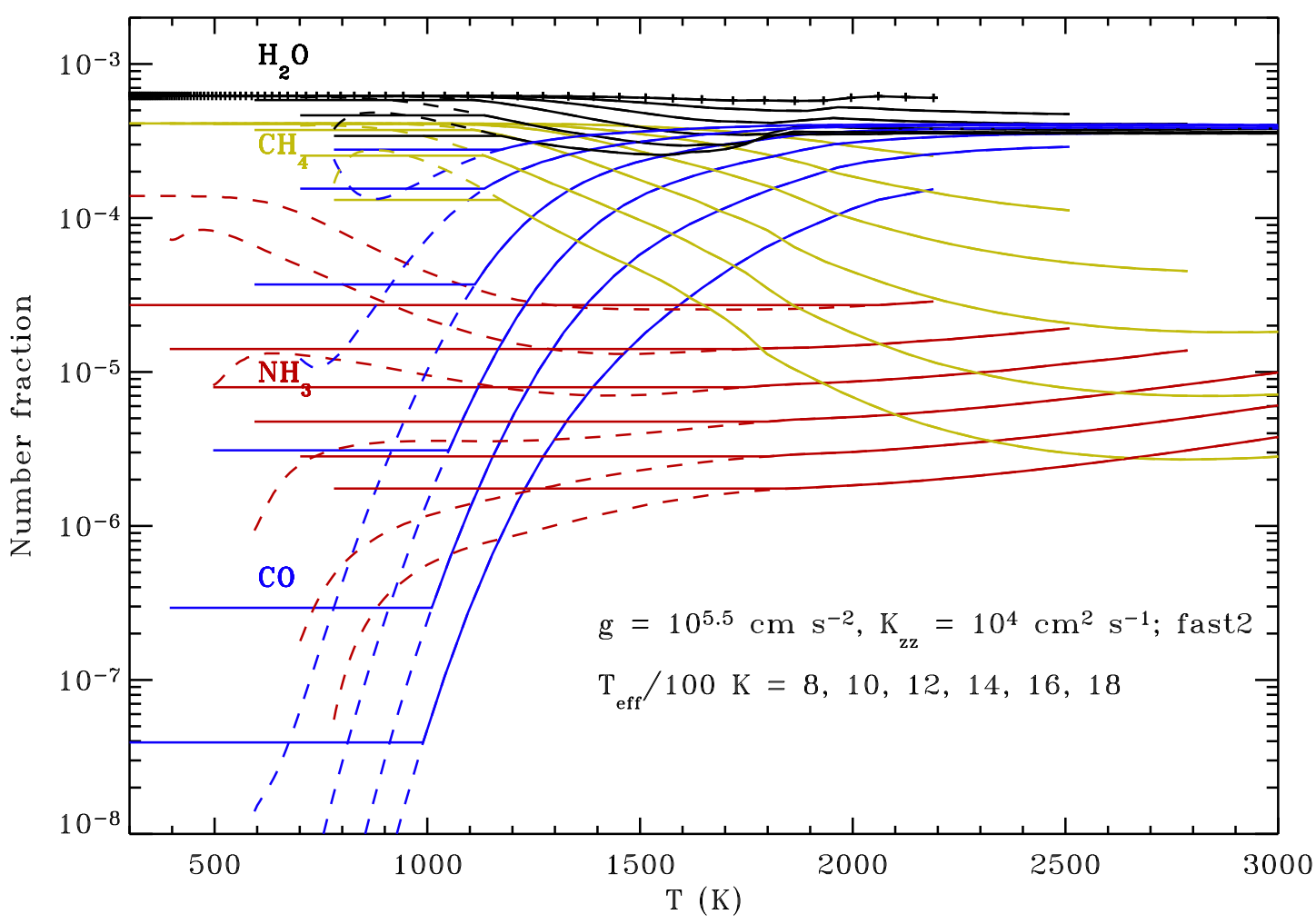

FIG. 2.- Similar to Fig. 1 but with a range of effective temperatures to display the trends with $T_{\text {eff }}$. The color scheme is the same as in Fig. 1 The association of the individual lines with effective temperatures is made by noting that in the plot the lines corresponding to progressively lower effective temperatures end at progressively lower local temperatures. (In other words, the longer the lines go to the right, the higher the effective temperature.) In all cases displayed here, water and methane remain at their equilibrium abundances. The degree of departure from equilibrium for ammonia decreases with increasing effective temperature. 


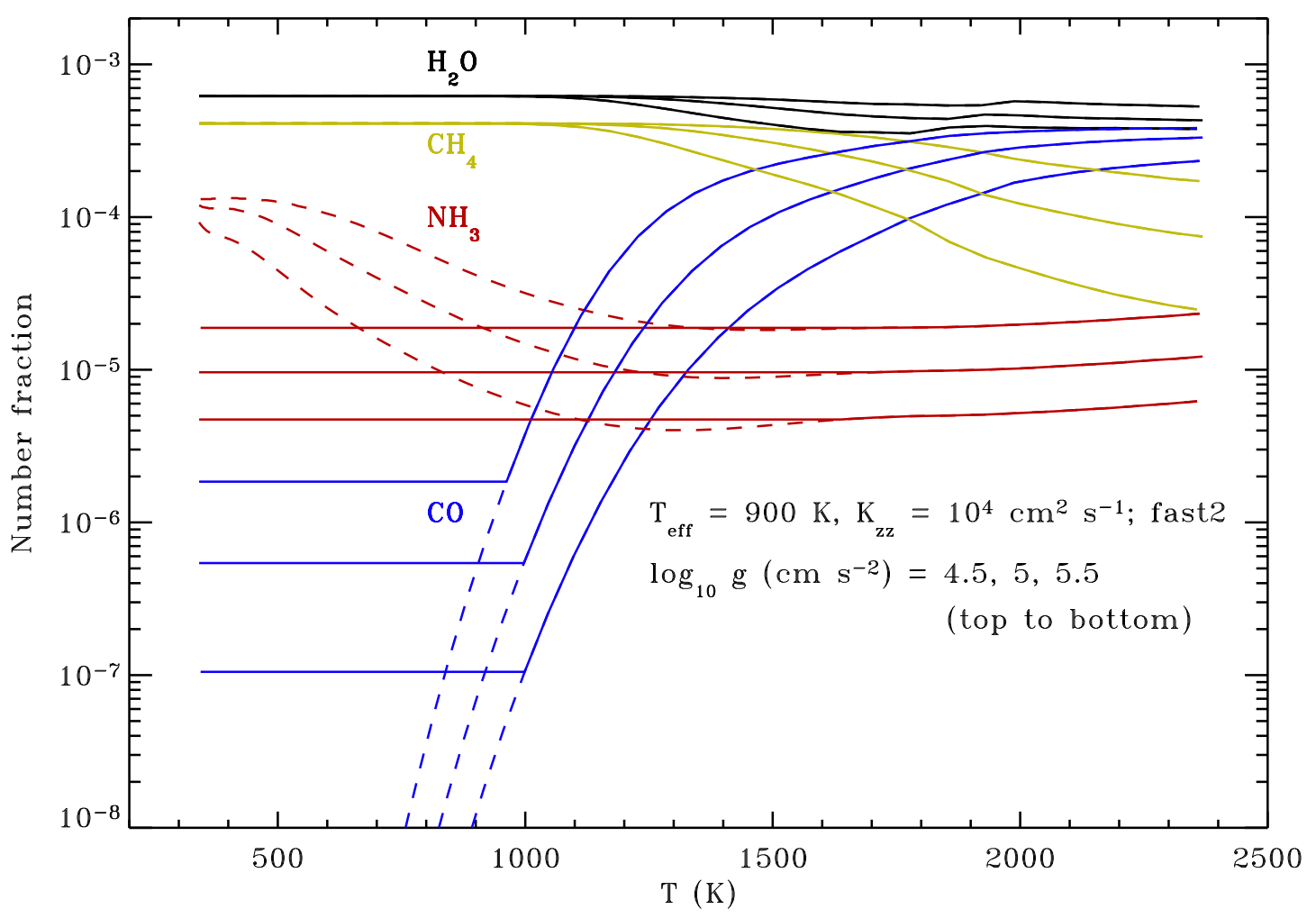

FIg. 3.- Similar to Fig. 2 but showing the trend with changing surface gravity. This figure demonstrates that the non-equilibrium abundance of $\mathrm{CO}$ increases with decreasing gravity. See explanation in the text. 


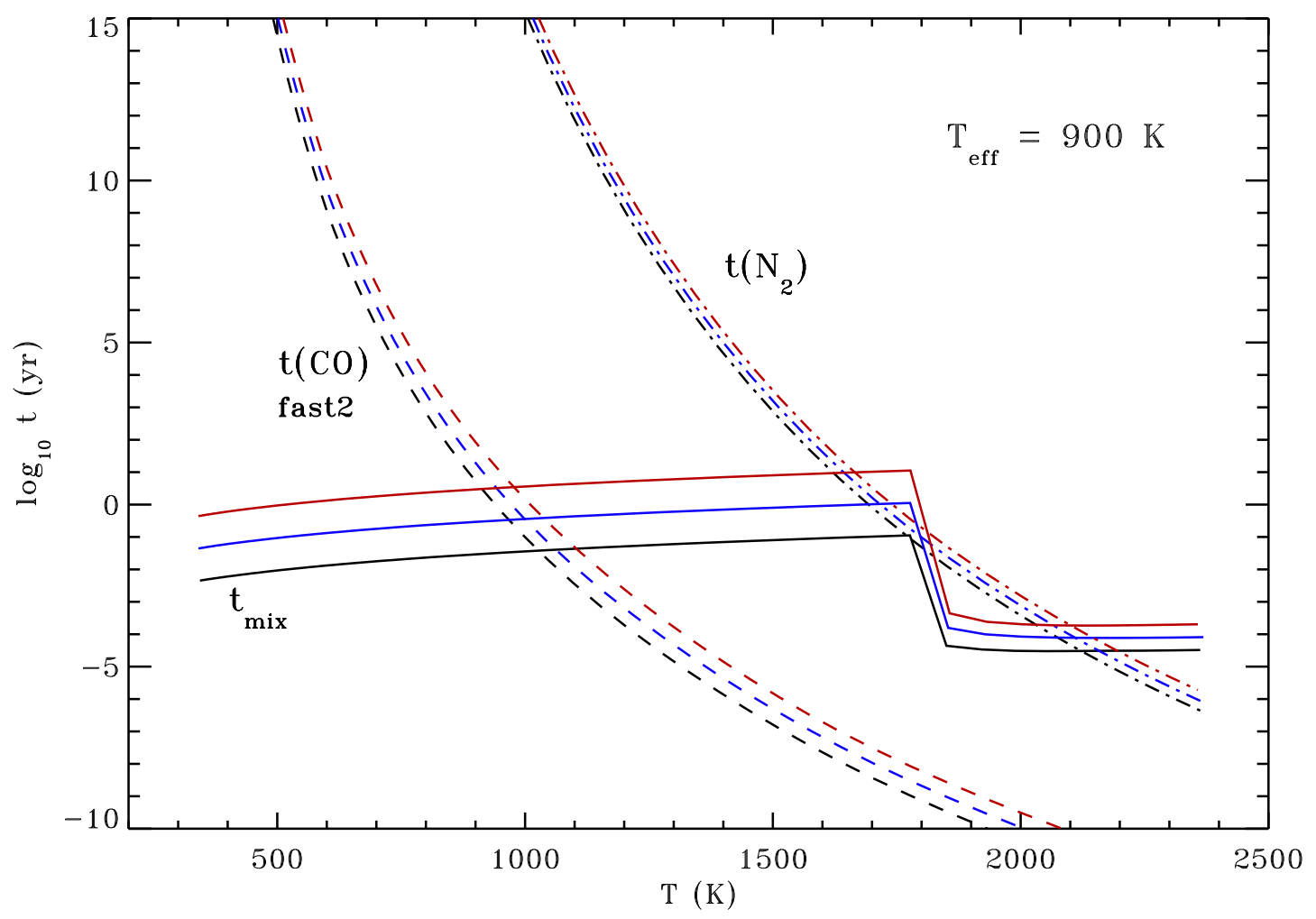

FIG. 4. - A plot of the mixing time (solid lines), the reaction time for the $\mathrm{CO} / \mathrm{CH}_{4}$ (dashed lines), and the reaction time for the $\mathrm{NH}_{3} / \mathrm{N}_{2}$ reaction (dash-dotted lines), for models with $T_{\text {eff }}=900 \mathrm{~K}, K_{z z}=10^{4} \mathrm{~cm}^{2} \mathrm{~s}^{-1}$, and $\log _{10} g\left(\mathrm{~cm} \mathrm{~s}^{-2}\right)=5.5$ (black), 5.0 (blue), and 4.5 (red). The abrupt change of the mixing-time curves represents the boundary of the convection zone. 


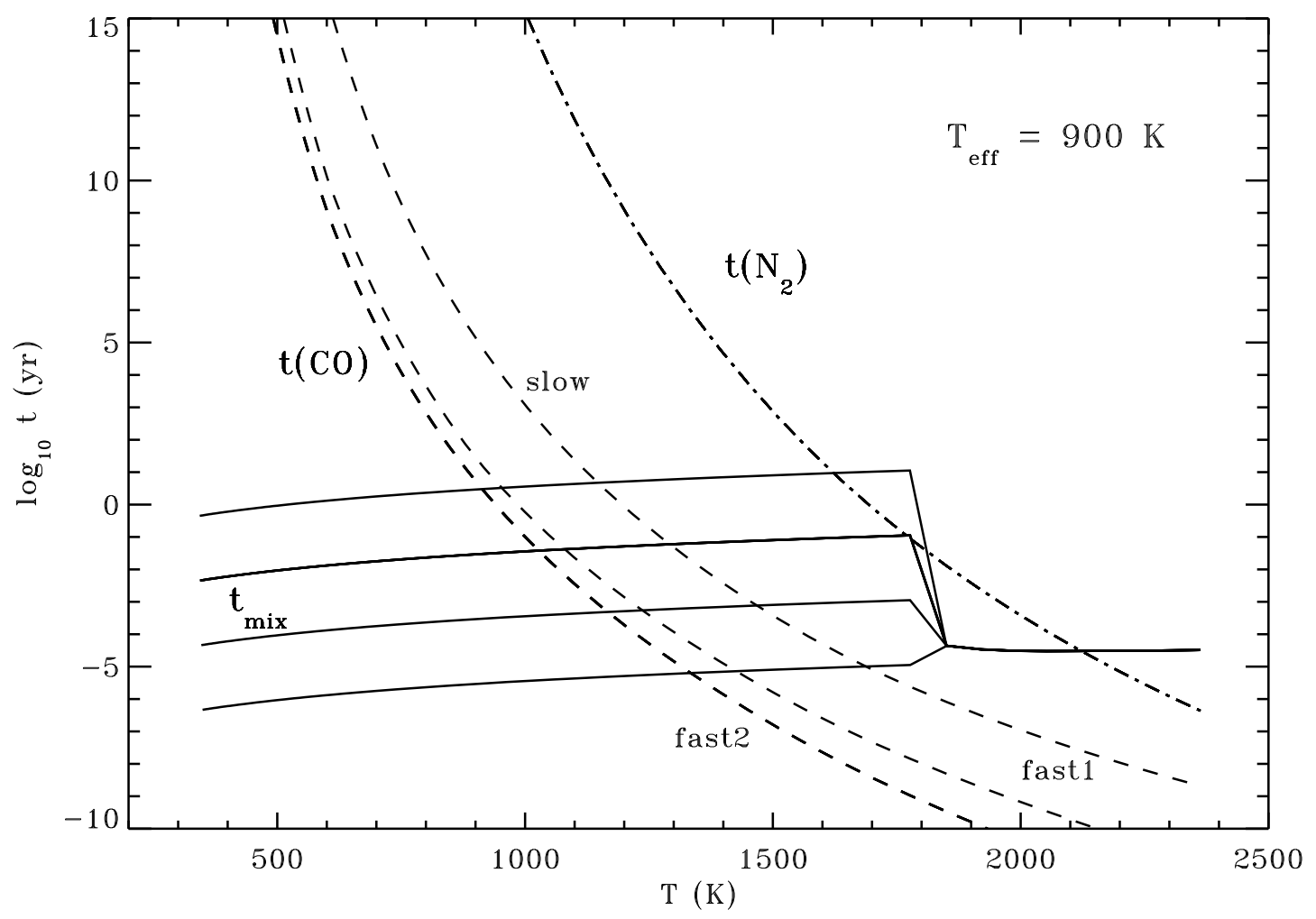

Fig. 5.- Similar to Fig. 4 but depicting the mixing times (solid lines) corresponding to (from top to bottom) the four values of the diffusion coefficient, $K_{z z}=10^{2}, 10^{4}, 10^{6}, 10^{8} \mathrm{~cm}^{2} \mathrm{~s}^{-1}$, for models with $T_{\text {eff }}=900 \mathrm{~K}$, and $\mathrm{g}=10^{5.5} \mathrm{~cm} \mathrm{~s}^{-2}$. Also shown are the reaction times for $\mathrm{CO} / \mathrm{CH}_{4}$ (dashed lines) chemistry, for the three different treatments of the reaction speed, "slow," "fast1," and "fast2" (the curves are labeled accordingly), and the reaction times for the $\mathrm{NH}_{3} / \mathrm{N}_{2}$ reaction (dot-dashed lines). 


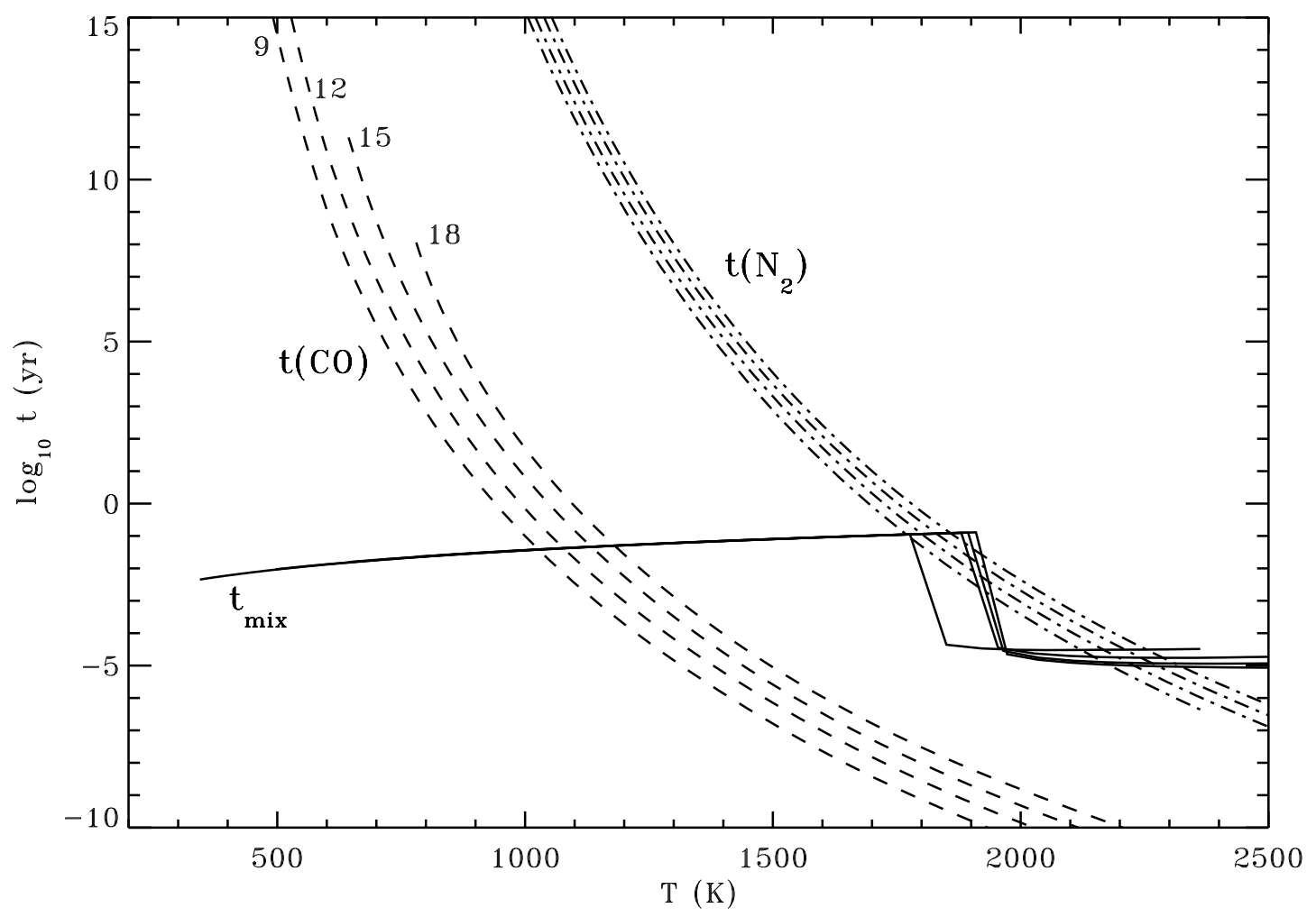

FIG. 6.- Similar to Fig. 4 but depicting the mixing times (solid lines), and the reaction times for CO/ $\mathrm{CH}_{4}$ (dashed lines) for the "fast2" reaction rate, and the reaction time for the $\mathrm{NH}_{3} / \mathrm{N}_{2}$ reaction (dot-dashed line), for a set of models with $\mathrm{g}=10^{5.5} \mathrm{~cm} \mathrm{~s}^{-2}, K_{z z}=10^{4} \mathrm{~cm}^{2}$ $\mathrm{s}^{-1}$, and $T_{\text {eff }} / 100 \mathrm{~K}=9,12,15,18$. The reaction times for $\mathrm{CO} / \mathrm{CH}_{4}$ are labeled accordingly. 


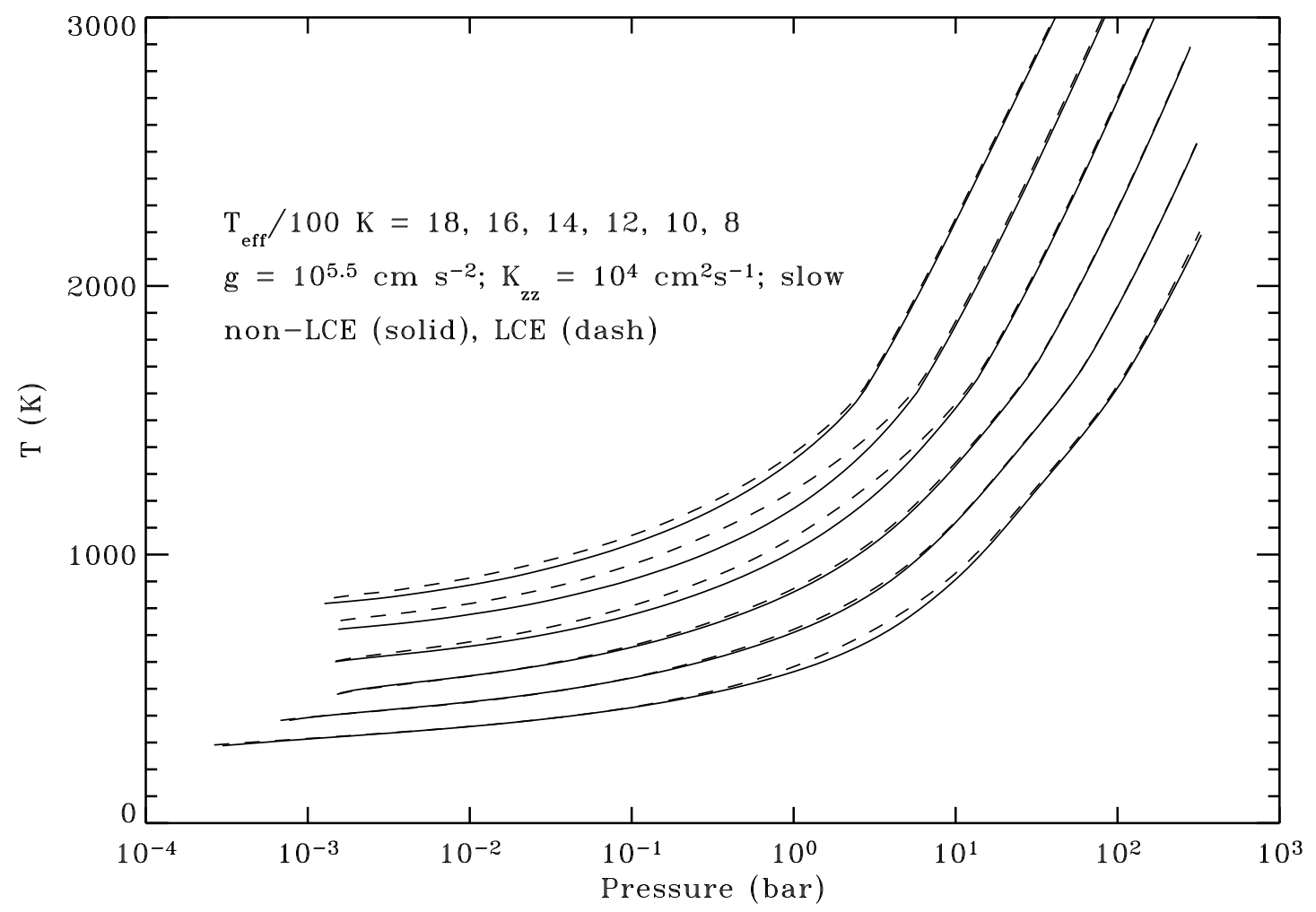

FIG. 7.- A comparison of temperature-pressure profiles for equilibrium (dashed lines) and non-equilibrium (solid lines) models of $T_{\text {eff }}=800,1000,1200,1400,1600$, and $1800 \mathrm{~K}$ (from bottom to top). $g$ is equal to $10^{5.5} \mathrm{~cm} \mathrm{~s}^{-2}$. 


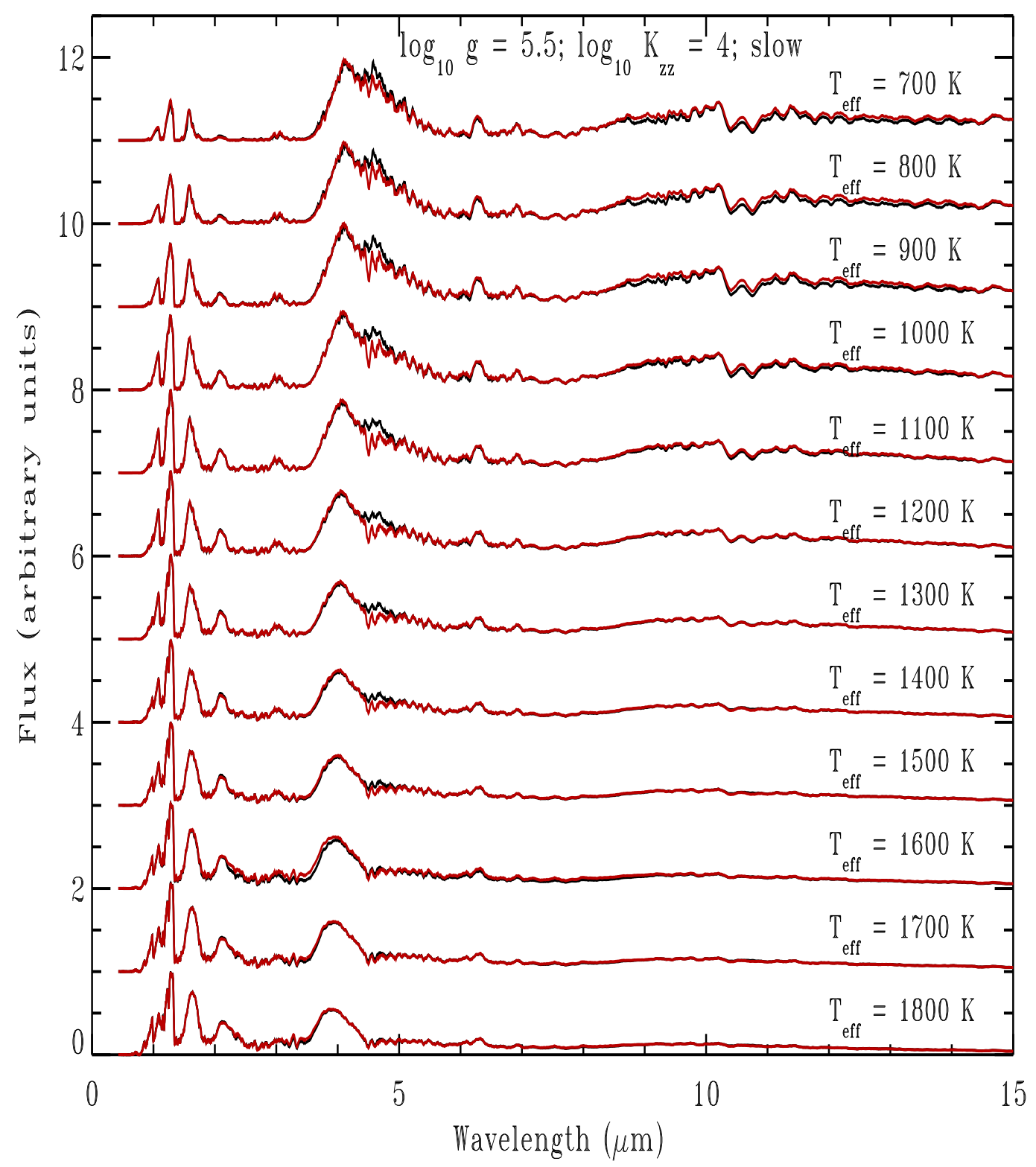

FIG. 8. - Synoptic comparison of model atmosphere spectra computed with chemical equilibrium (black lines), and with departures from equilibrium (red lines), for a representative value of $g=10^{5.5} \mathrm{~cm} \mathrm{~s}^{-2}$, and $K_{z z}=10^{4} \mathrm{~cm}^{2} \mathrm{~s}^{-1}$, for a "slow" $\mathrm{CO} / \mathrm{CH}_{4}$ reaction, and for effective temperatures between 700 and $1800 \mathrm{~K}$, covering most of the T and L spectral types. There are essentially two wavelength regions where the departure from chemical equilibrium influences the predicted spectrum: the region around $4.7 \mu \mathrm{m}$ (due to non-equilibrium abundances of $\mathrm{CO}$ ), and a wide region between 8 and $14 \mu \mathrm{m}$, due to non-equilibrium abundances of $\mathrm{NH}_{3}$. The $\mathrm{CO}$ feature has its peak for effective temperatures between $800 \mathrm{~K}$ and $1200 \mathrm{~K}$; the effect generally decreases for hotter models and essentially disappears at $1800 \mathrm{~K}$. The figure is similar to that presented by Saumon et al. (2003). 


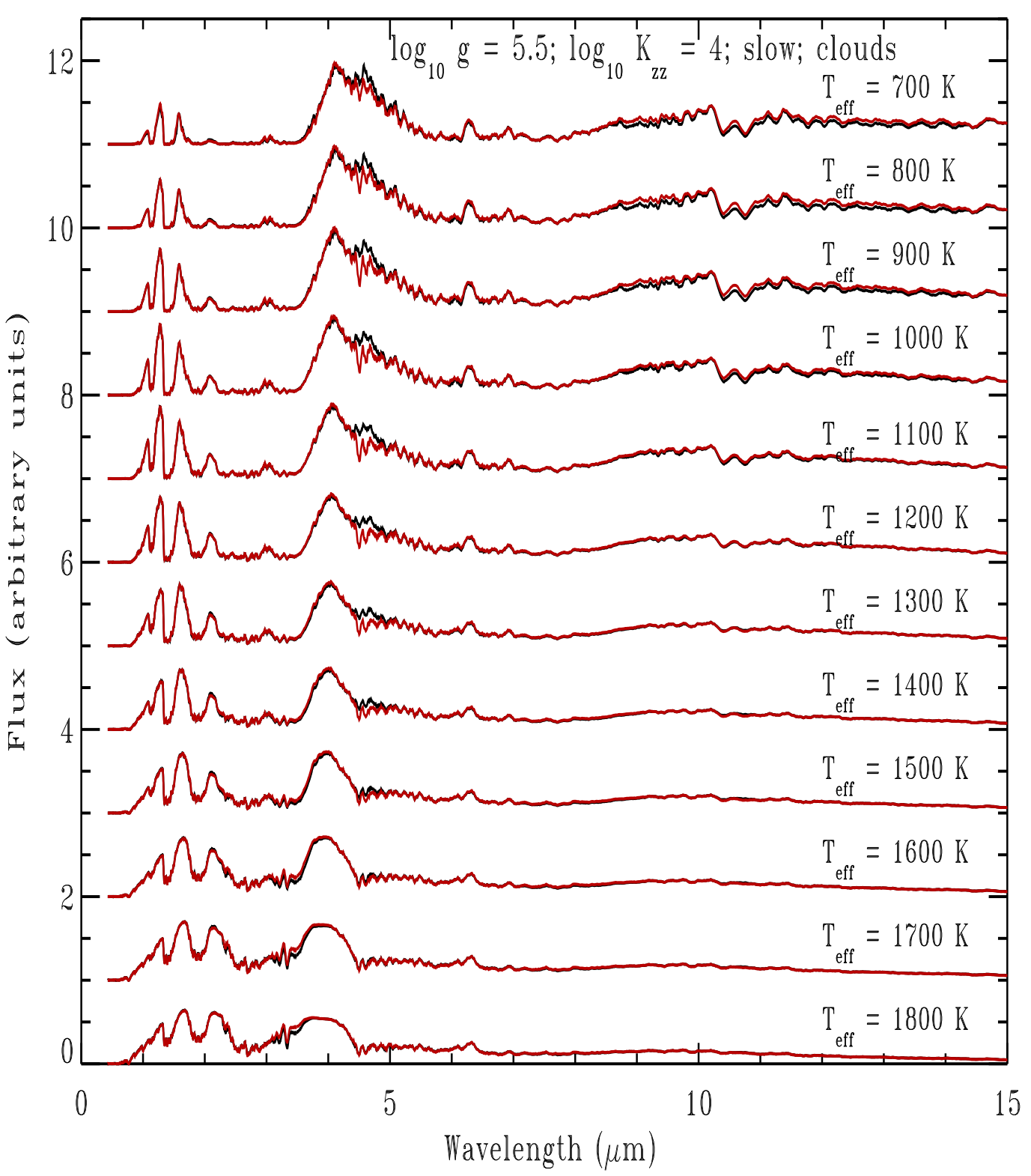

FIG. 9. - The same as Fig. 8 the only difference is that the models are computed with clouds. We assume a cloud composed of a collection of magnesium silicate componds represented by a single species, in this case forsterite, with an extension of the cloud layer toward higher pressures, as suggested by BSH (E-type cloud model in their notation). We assume a modal particle size of $100 \mu \mathrm{m}$. The presence of clouds leads to differences in the predicted spectrum, in particular for wavelengths below $2.5 \mu \mathrm{m}$, but otherwise the effect of departures from chemical equilibrium is essentially the same as for the cloudless models. 


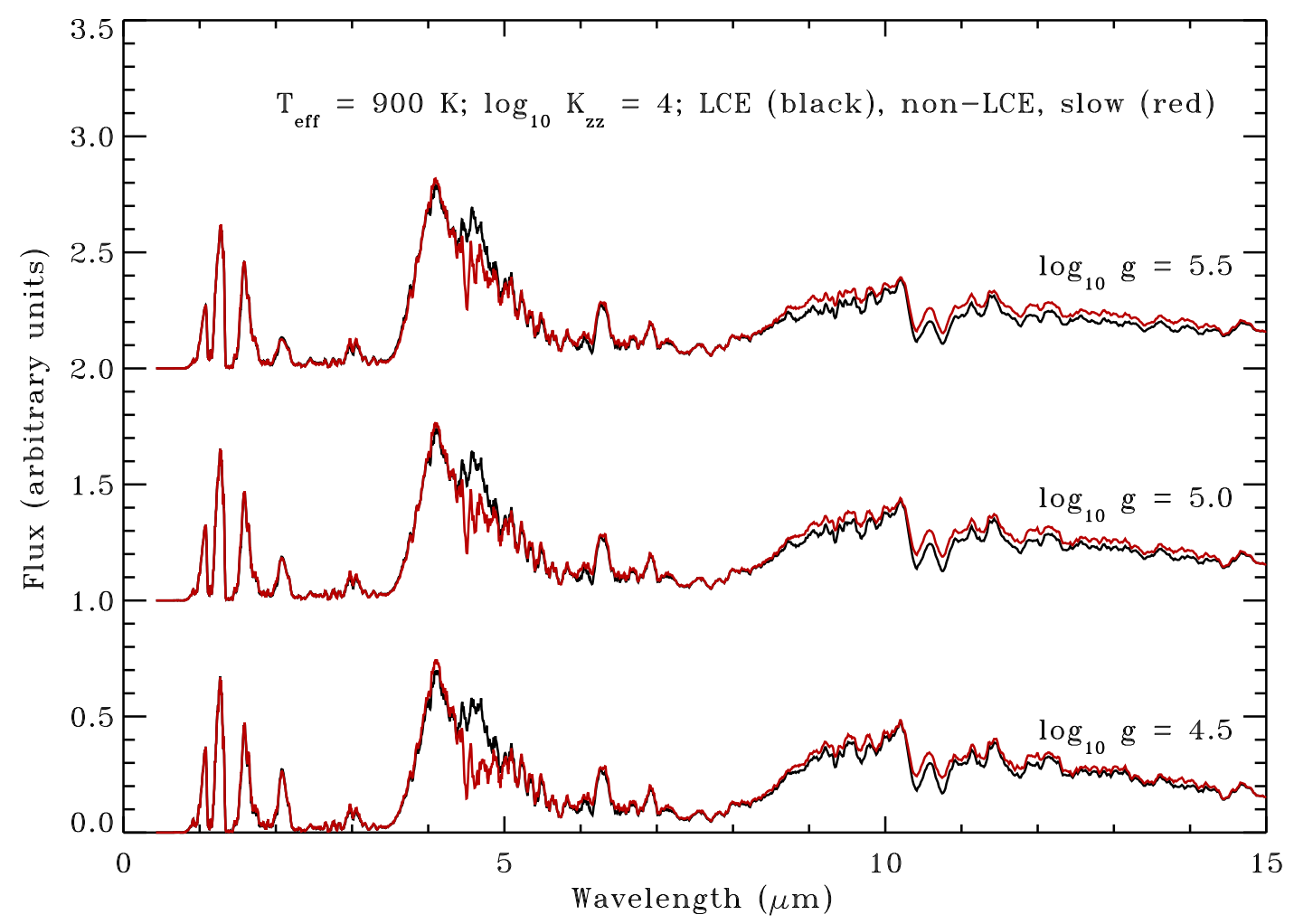

FIG. 10.- A comparison of predicted spectra for a model with $T_{\text {eff }}=900 \mathrm{~K}, K_{z z}=10^{4} \mathrm{~cm}^{2} \mathrm{~s}^{-1}$, and three different surface gravities. We display the equilibrium models (black lines), and non-equilibrium models (red lines) assuming the "slow" $\mathrm{CO} / \mathrm{CH}_{4}$ reaction rate of Prinn \& Barshay (1977). The plot also clearly shows that the effects of departures from chemical equilibrium are more pronounced for lower gravities. See Fig. 1 and the text for explanations. 


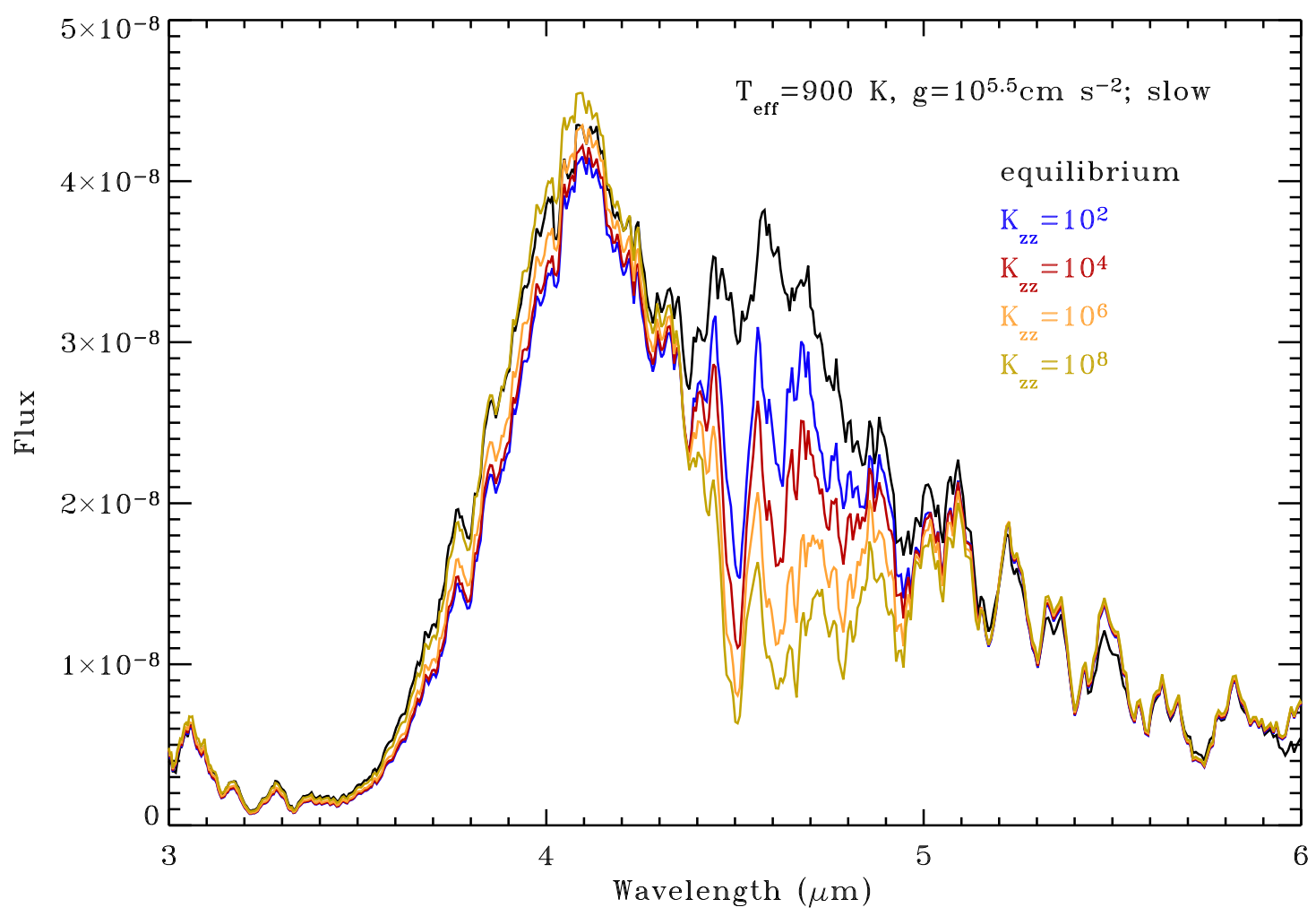

FIG. 11. - A more detailed view of the CO non-equilibrium region between 3 and $5 \mu \mathrm{m}$, for models with $T_{\text {eff }}=900 \mathrm{~K}$ and $g=10^{5.5} \mathrm{~cm}$ $\mathrm{s}^{-2}$. Displayed are the equilibrium model (black), as well four non-equilibrium models with $K_{z z}=10^{2}$ (blue), $10^{4}$ (red), $10^{6}$ (yellow), and $10^{8}$ (green) $\mathrm{cm}^{2} \mathrm{~s}^{-1}$. All models are for the "slow" reaction rate for the $\mathrm{CO} / \mathrm{CH}_{4}$ reaction of Prinn \& Barshay (1977). 


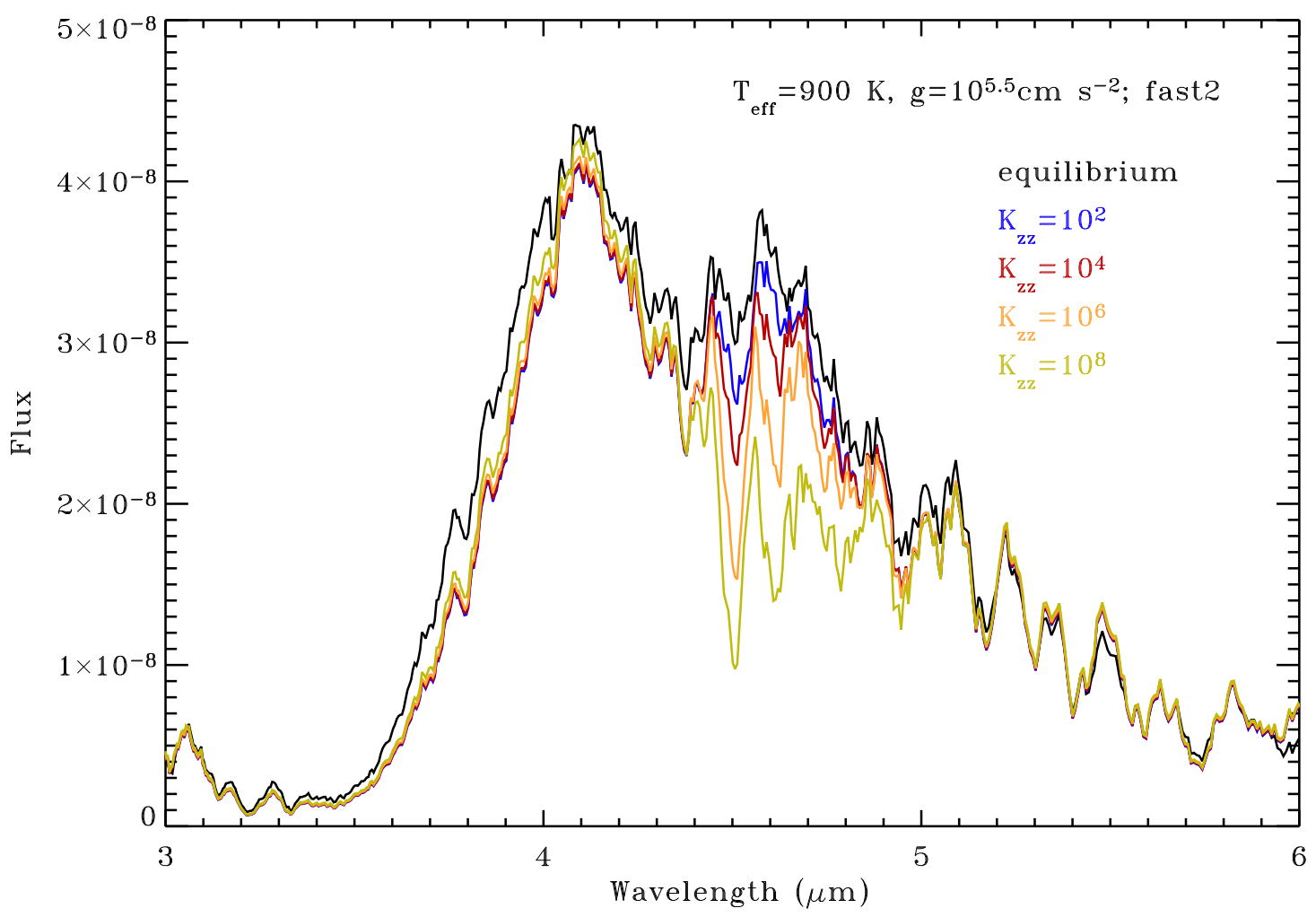

FIG. 12.- Similar to Fig. 11 but for the "fast2" $\mathrm{CO} / \mathrm{CH}_{4}$ reaction rate of Yung et al. (1988). Departures from equilibrium are now smaller, as explained in the text. 


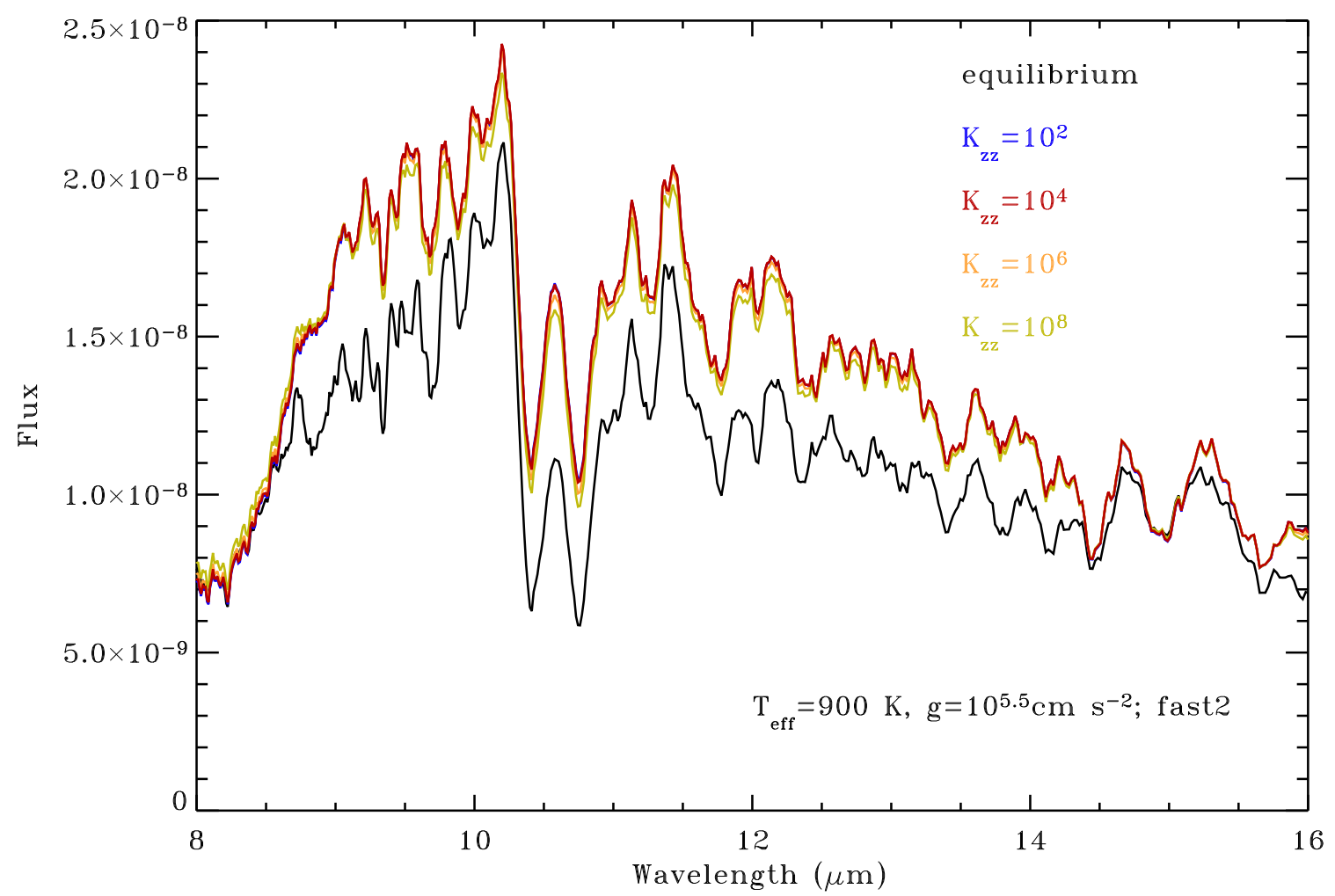

FIG. 13. - A more detailed view of the ammonia region between 8 and $14 \mu \mathrm{m}$, for the same models as displayed in Fig. 12 The curves for all non-equilibrium models essentially coincide, except for the highest value of $K_{z z}=10^{8} \mathrm{~cm}^{2} \mathrm{~s}^{-1}$. The flux in the region between 8 and $14 \mu \mathrm{m}$ is relatively insensitive to the value of $K_{z z}$. 

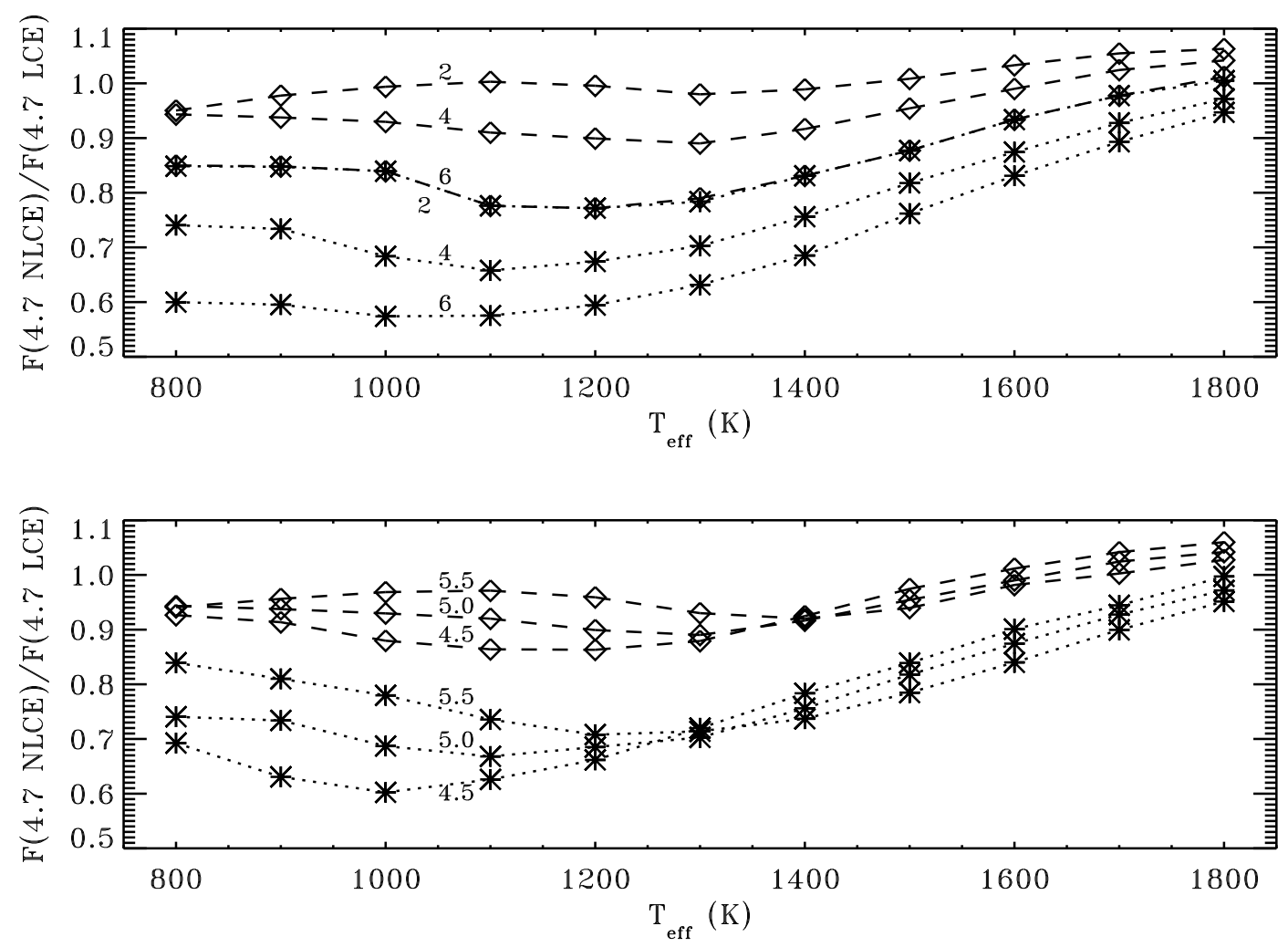

FIG. 14.- Departures from chemical equilibrium in the 4.7- $\mu \mathrm{m}$ CO region. The plot displays the ratio of the integrated flux in the region between $4.4 \mu \mathrm{m}$ and $5 \mu \mathrm{m}$ of the non-equilibrium and equilibrium models, as a function of effective temperature. The upper panel displays the dependence of the non-equilibrium ratio for $g=10^{5} \mathrm{~cm} \mathrm{~s}^{-2}$, for the "slow" $\mathrm{CO} / \mathrm{CH}_{4}$ reaction (the upper set of diamonds connected by dashed lines) and the "fast2" $\mathrm{CO} / \mathrm{CH}_{4}$ reaction (the lower set of stars connected by dotted lines); each set is shown for three values of $K_{z z}$. The curves are labelled by the value of $\log _{10} K_{z z}$ in $\mathrm{cm}^{2} \mathrm{~s}^{-1}$. The curves for $K_{z z}=10^{6} \mathrm{~cm}^{2} \mathrm{~s}^{-1}$ for the "fast2" $\mathrm{CO} / \mathrm{CH}_{4}$ reaction and for $K_{z z}=10^{2} \mathrm{~cm}^{2} \mathrm{~s}^{-1}$ for the "slow" $\mathrm{CO} / \mathrm{CH}_{4}$ reaction essentially coincide. The lower panel displays the dependence of the non-equilibrium ratio on surface gravity for two sets of models. In all models, $K_{z z}=10^{4} \mathrm{~cm}^{2} \mathrm{~s}^{-1}$. The upper set of models (diamonds connected by dashed lines) are for the "fast2" $\mathrm{CO} / \mathrm{CH}_{4}$ reaction, while the lower set of models (stars connected by dotted lines) are for the "slow" $\mathrm{CO} / \mathrm{CH}_{4}$ reaction. The curves are labelled by the value of $\log _{10} g$ in $\mathrm{cm} \mathrm{s}^{-2}$. 


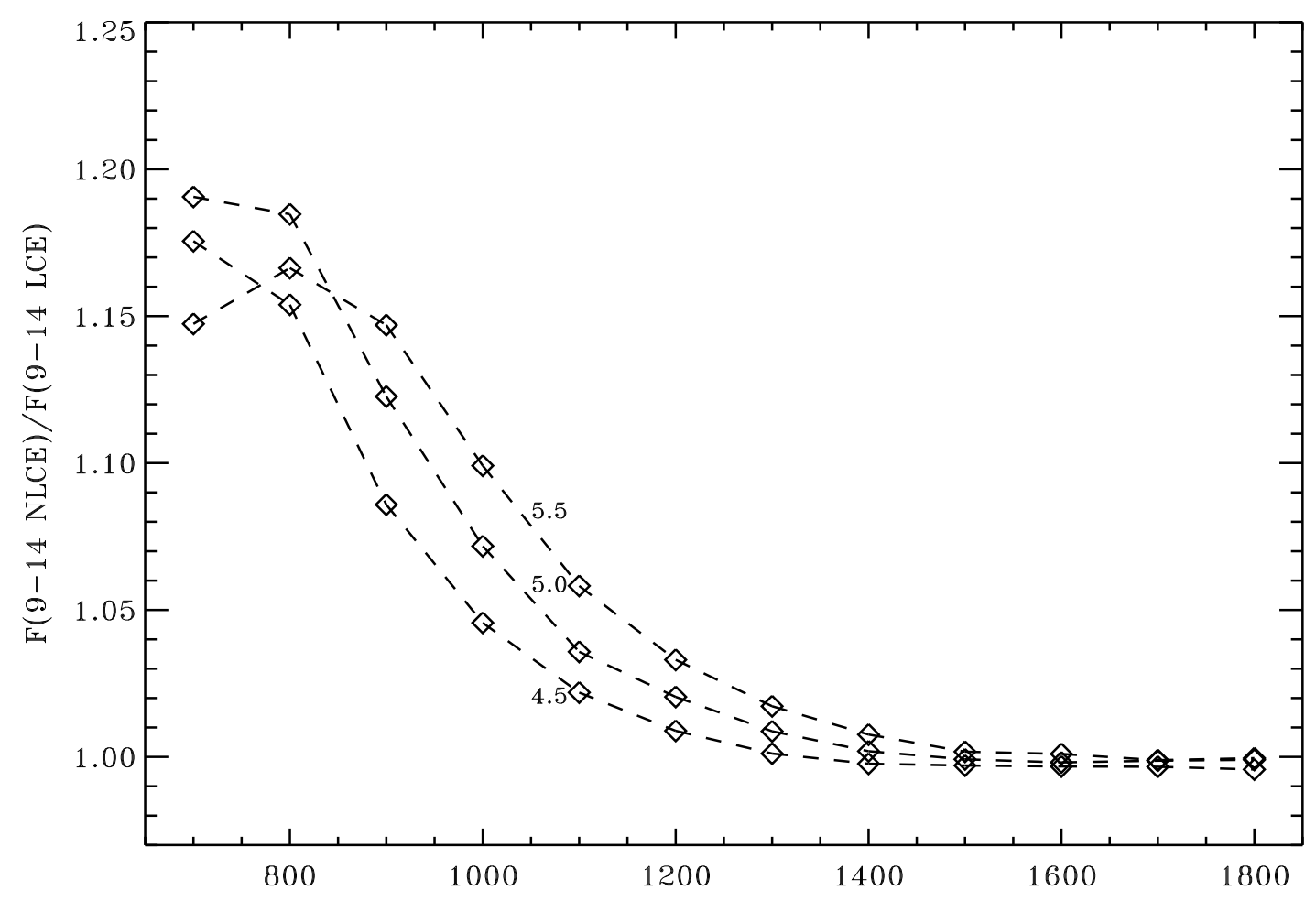

FIG. 15. - Similar to Fig. 14 but for the ammonia region between $9 \mu \mathrm{m}$ and $14 \mu \mathrm{m}$. Since the predicted spectra are insensitive to the speed of the $\mathrm{CO} / \mathrm{CH}_{4}$ reaction, and only weakly sensitive to $K_{z z}$, we display only models with $K_{z z}=10^{4}$, and the "fast2" $\mathrm{CO} / \mathrm{CH} 4$ reaction. The curves are labelled by the value of $\log _{10} g$ in $\mathrm{cm} \mathrm{s}^{-2}$. 


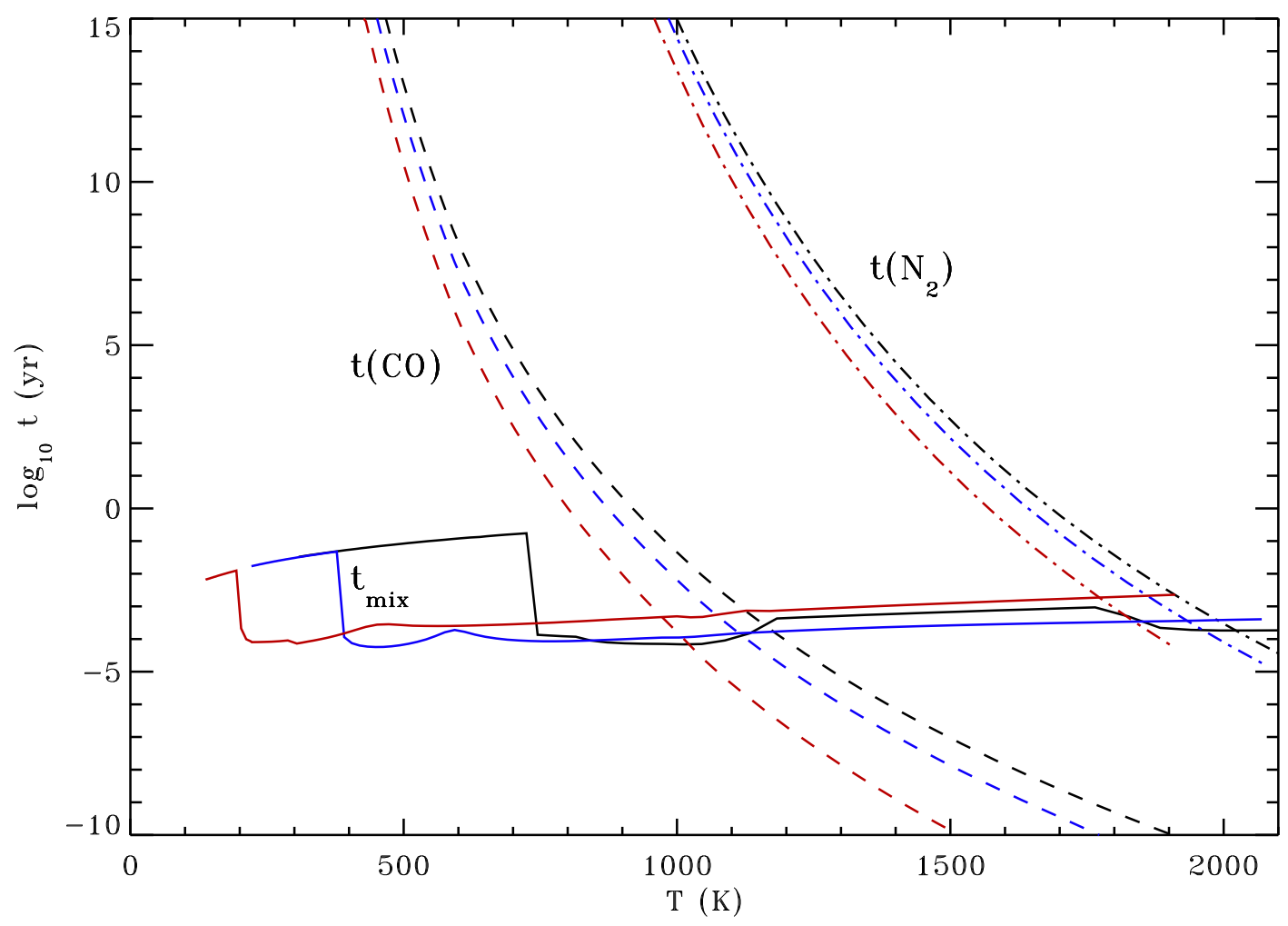

FIG. 16. - A plot of the mixing time (solid lines), the reaction time for the $\mathrm{CO} / \mathrm{CH}_{4}$ (dashed lines), and the reaction time for the $\mathrm{NH} 3 / \mathrm{N}_{2}$ reaction (dash-dotted lines), for models with $g=10^{5} \mathrm{~cm} \mathrm{~s}^{-2} K_{z z}=10^{4} \mathrm{~cm}^{2} \mathrm{~s}^{-1}$, and $T_{\text {eff }}=600 \mathrm{~K}$ (black lines), $400 \mathrm{~K}$ (blue lines), and $200 \mathrm{~K}$ (red lines). 


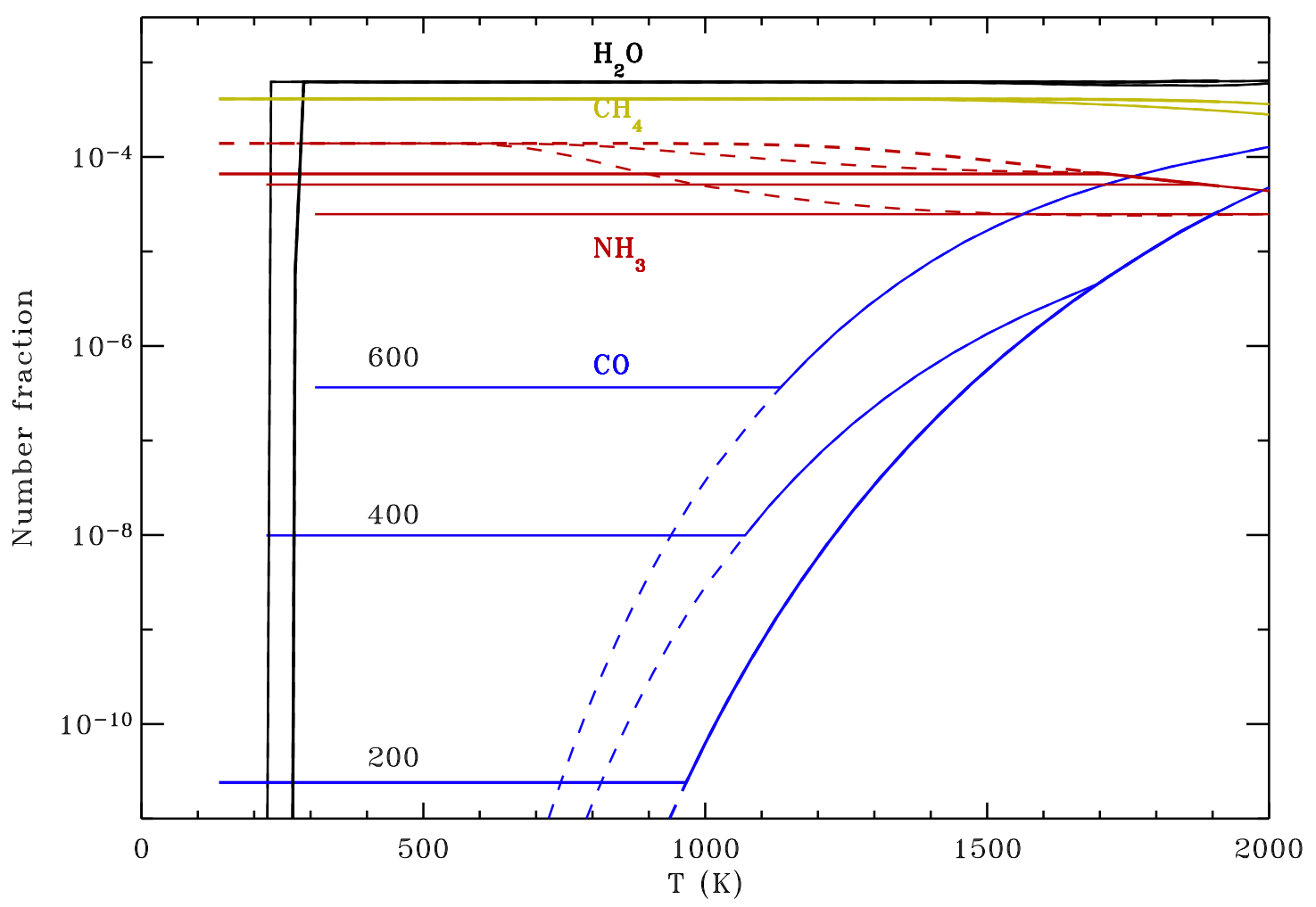

FIG. 17.- A comparison of the equilibrium (dashed lines) and non-equilibrium (solid lines) abundances of water (black), methane (yellow), ammonia (red), and CO (blue), for the same models as displayed in Fig. 16 Thin lines depict models for $T_{\text {eff }}=600 \mathrm{~K}$, thicker lines for for $T_{\text {eff }}=400 \mathrm{~K}$, and the thickest lines for $T_{\text {eff }}=200 \mathrm{~K}$. The curves for CO are labeled by the value of $T_{\text {eff }}$ in K. 

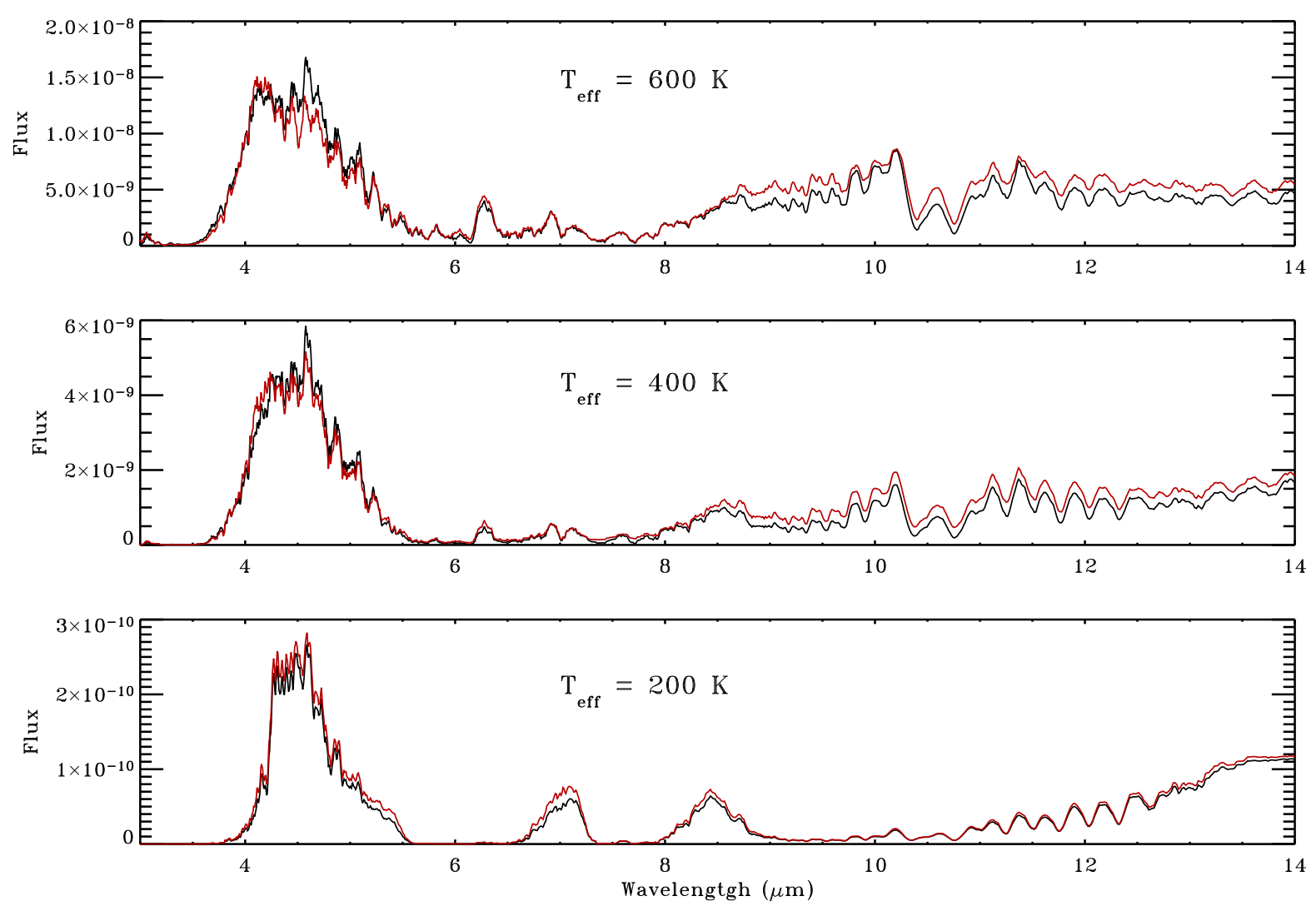

FIG. 18. - Predicted flux for models with equilibrium (black) and non-equilibrium (red) models with $g=10^{5} \mathrm{~cm} \mathrm{~s}^{-2} K_{z z}=10^{4} \mathrm{~cm}^{2}$ $\mathrm{s}^{-1}$, and $T_{\text {eff }}=600 \mathrm{~K}$ (upper panel), $400 \mathrm{~K}$ (middle panel), and $200 \mathrm{~K}$ (lower panel). 


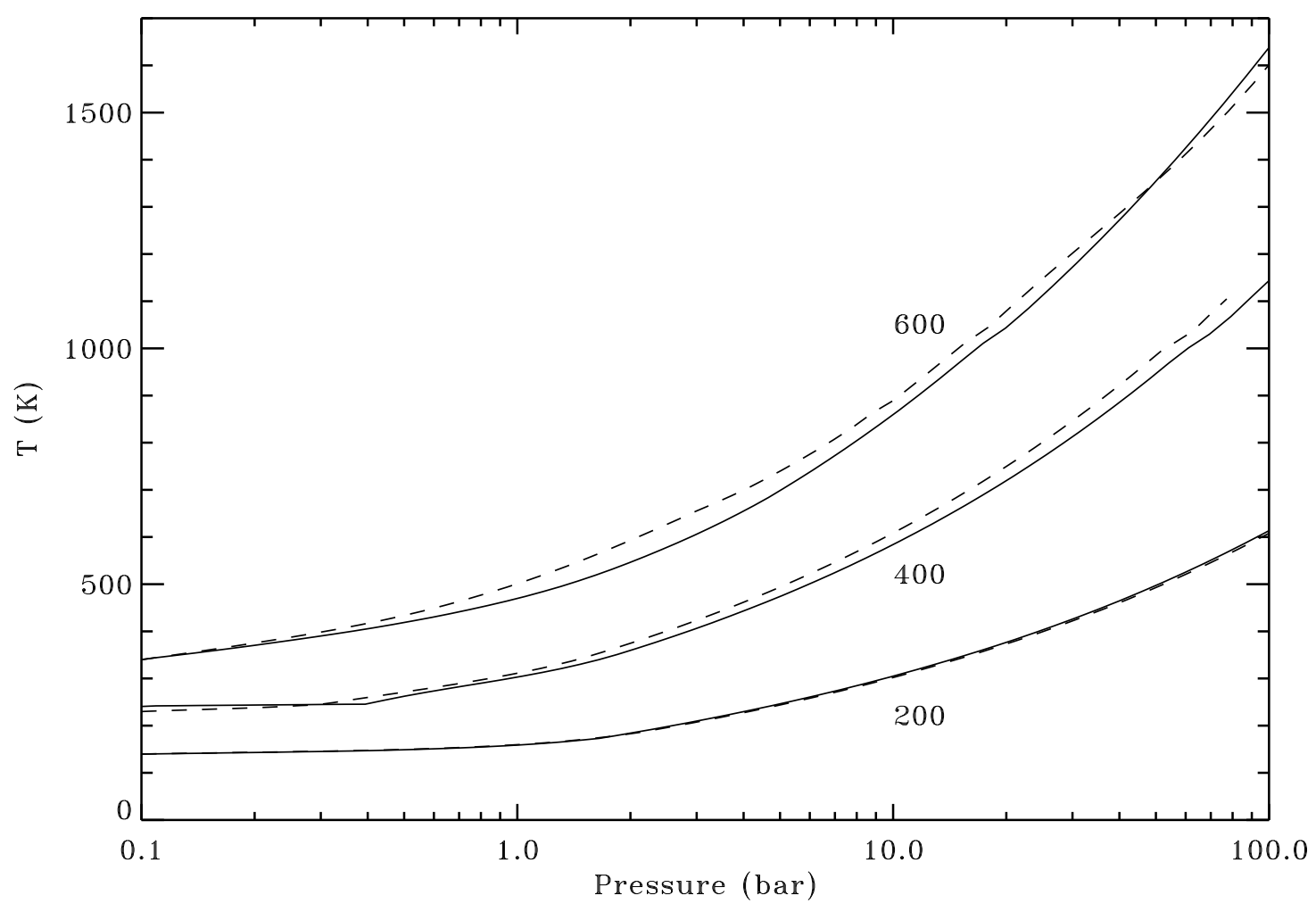

FIG. 19.- A comparison of temperature-pressure profiles for equilibrium (dashed lines) and non-equilibrium (solid lines) models of $T_{\text {eff }}=600,400$, and $200 \mathrm{~K}$ (from top to bottom). $g$ is equal to $10^{5} \mathrm{~cm} \mathrm{~s}^{-2}$. 


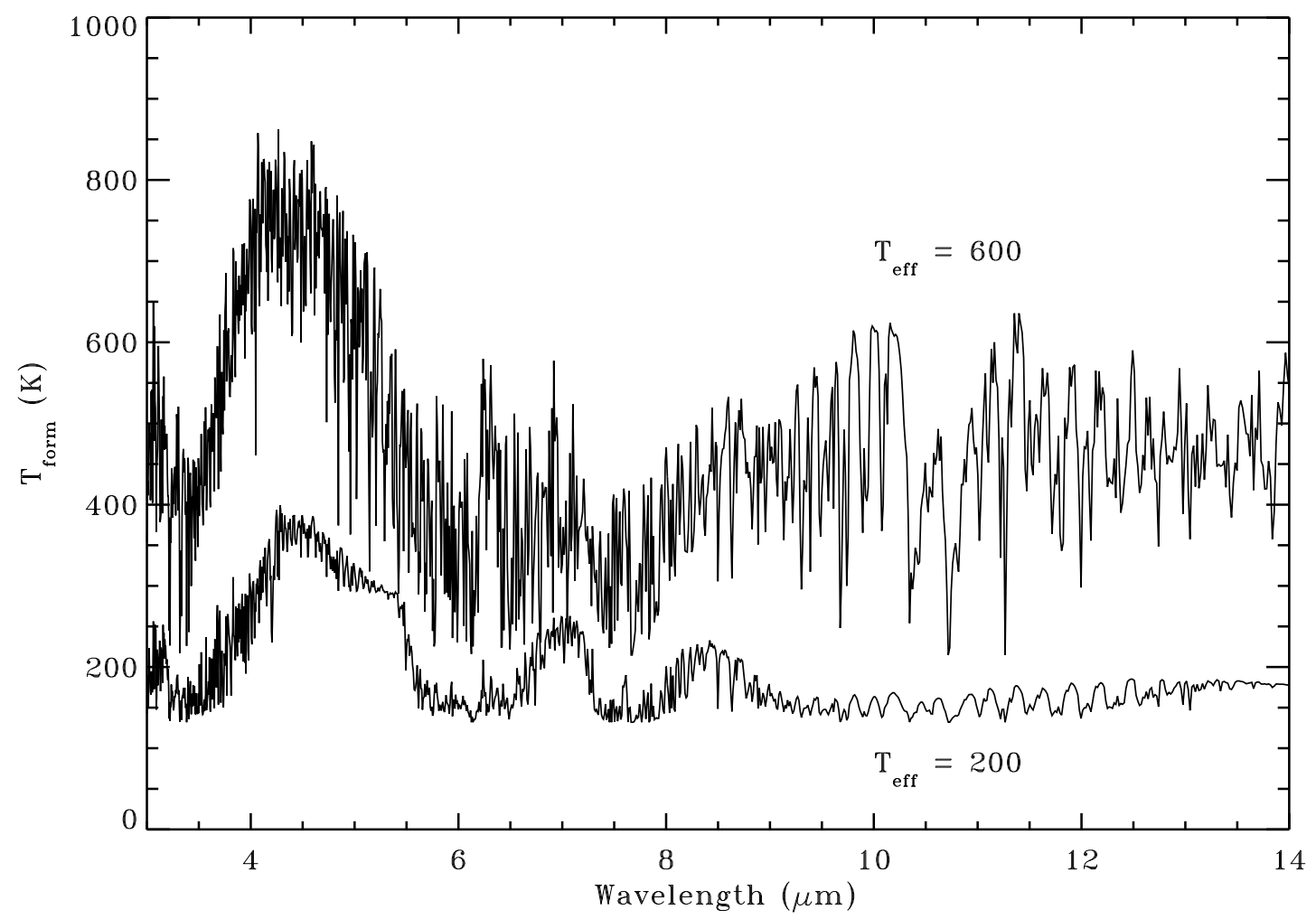

FIG. 20.- Radiation formation temperature as a function of wavelength for models with $T_{\text {eff }}=600 \mathrm{~K}$ (upper curve) and $200 \mathrm{~K}$ (lower curve). $g$ is equal to $10^{5} \mathrm{~cm} \mathrm{~s}^{-2}$. 


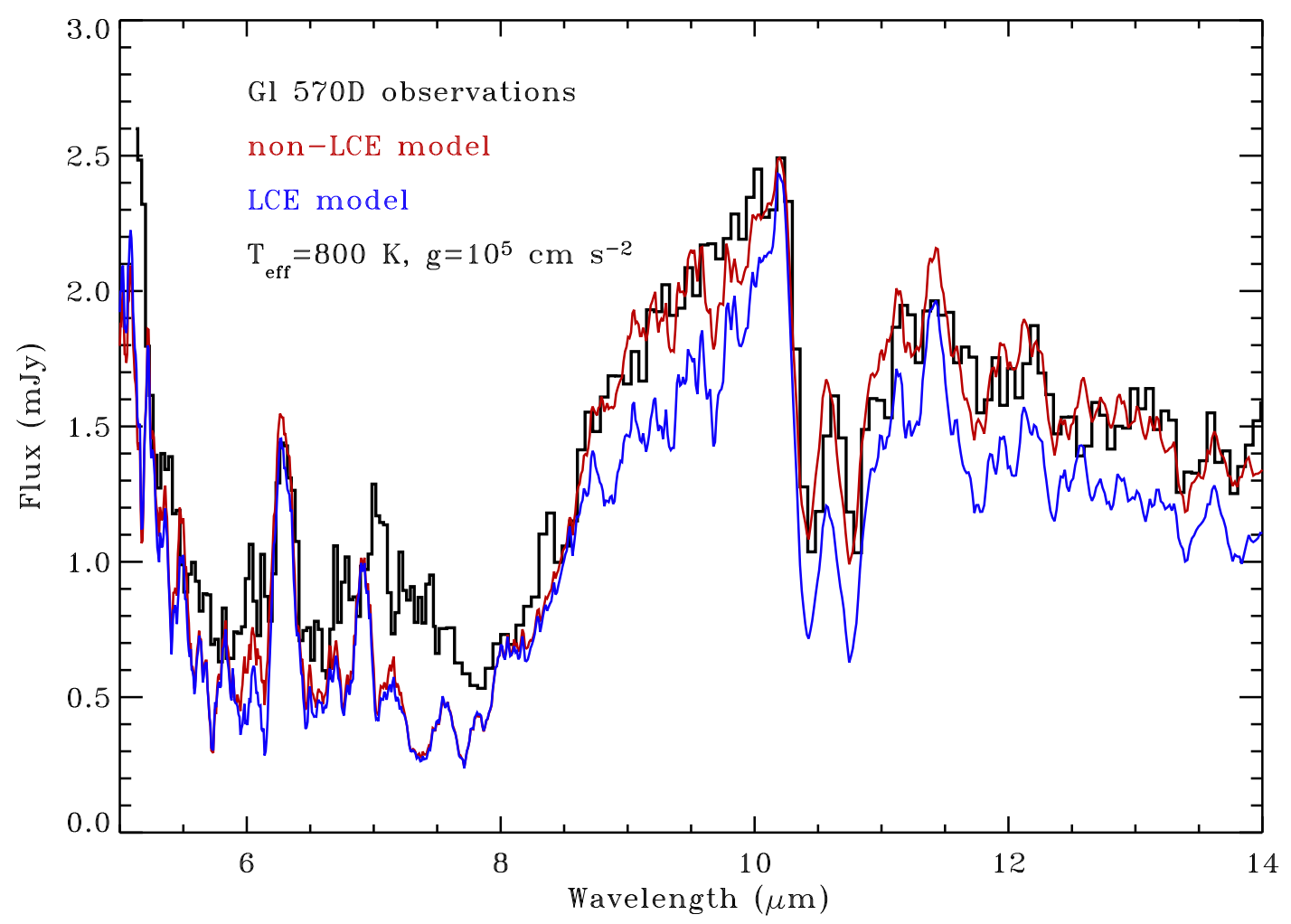

FIG. 21. - Comparison of observed and predicted spectra of Gl 570D. Thick black histogram displays the observed flux, the red and blue lines are the predicted spectra for non-equilibrium (red) and equilibrium (blue) models, computed for $T_{\text {eff }}=800 \mathrm{~K}$ and $\mathrm{g}=10^{5} \mathrm{~cm} \mathrm{~s}^{-2}$. The non-equilibrium model displayed is computed with $K_{z z}=10^{4} \mathrm{~cm}^{2} \mathrm{~s}^{-1}$, and for fast $\mathrm{CO} / \mathrm{CH}_{4}$ reaction rate, but, as demonstrated in Fig. 13 this predicted flux essentially coincides with the flux computed for all other values of $K_{z z}$ and $\mathrm{CO} / \mathrm{CH}_{4}$ reaction time prescriptions. 